\title{
Barrier kinetics of adsorption-desorption of alcohol monolayers on water under constant surface tension
}

\author{
Ivan L. Minkov, ${ }^{\mathrm{a}, \mathrm{b}}$ Dimitrinka Arabadzhieva, ${ }^{\mathrm{c}}$ Ibrahim E. Salama, ${ }^{\mathrm{d}, \mathrm{e}}$ \\ Elena Mileva, ${ }^{\mathrm{c}}$ Radomir I. Slavchov*,f,g \\ ${ }^{a}$ Department of Physical Chemistry, Faculty of Chemistry and Pharmacy, Sofia University, $1 \mathrm{~J}$. \\ Bourchier Blvd., 1164 Sofia, Bulgaria \\ ${ }^{\mathrm{b}}$ Department of Chemistry, Biochemistry, Physiology, and Pathophysiology, Faculty of Medicine, \\ Sofia University, 1 Koziak Str., 1407 Sofia, Bulgaria \\ ${ }^{\mathrm{c}}$ Bulgarian Academy of Science, Akad. G. Bonchev Str., bl.11, 1113 Sofia, Bulgaria, Sofia, Bulgaria \\ ${ }^{\mathrm{d}}$ Department of Chemisty, Cambridge University, CB2 1EW Cambridge, United Kingdom \\ ${ }^{\mathrm{e}}$ BP Institute, Cambridge University, Bullard Laboratories, Madingley Road, CB3 OEZ Cambridge, \\ United Kingdom \\ ${ }^{\mathrm{f}}$ Department of Chemical Engineering and Biotechnology, Cambridge University, Philippa Fawcett \\ Drive, West Site, CB3 OAS Cambridge, United Kingdom \\ ${ }^{\mathrm{g}}$ School of Engineering and Materials Science, Queen Mary University of London, Mile End Road, \\ London E1 4NS, United Kingdom \\ *E-mail: ris26@cam.ac.uk
}

\begin{abstract}
The desorption of spread decanol and dodecanol monolayers at controlled constant surface tension is shown to proceed under mixed barrier-diffusion control; the role of the convective diffusion is also discussed. The desorption rate is measured as a function of the density of the monolayer and the temperature. The rate of barrier desorption increases as the monolayer approaches the collapse point, reaching an infinite value. The average desorption time of an adsorbed dodecanol molecule increases linearly with the area per molecule, and is phase-specific - it is higher for the liquid condensed state of the monolayer than for the liquid expanded. The desorption rate increases with temperature; the activation energy for desorption is independent of the compression and the surface phase. The increase of the intensity of convection is shown to produce a vanishingly thin diffusion layer and causes the desorption to proceed under pure barrier control. A schematic map of the adsorption-desorption regimes acting as function of time and intensity of the convection is constructed. General expressions for the rate of adsorption and desorption of alcohols are formulated.
\end{abstract}




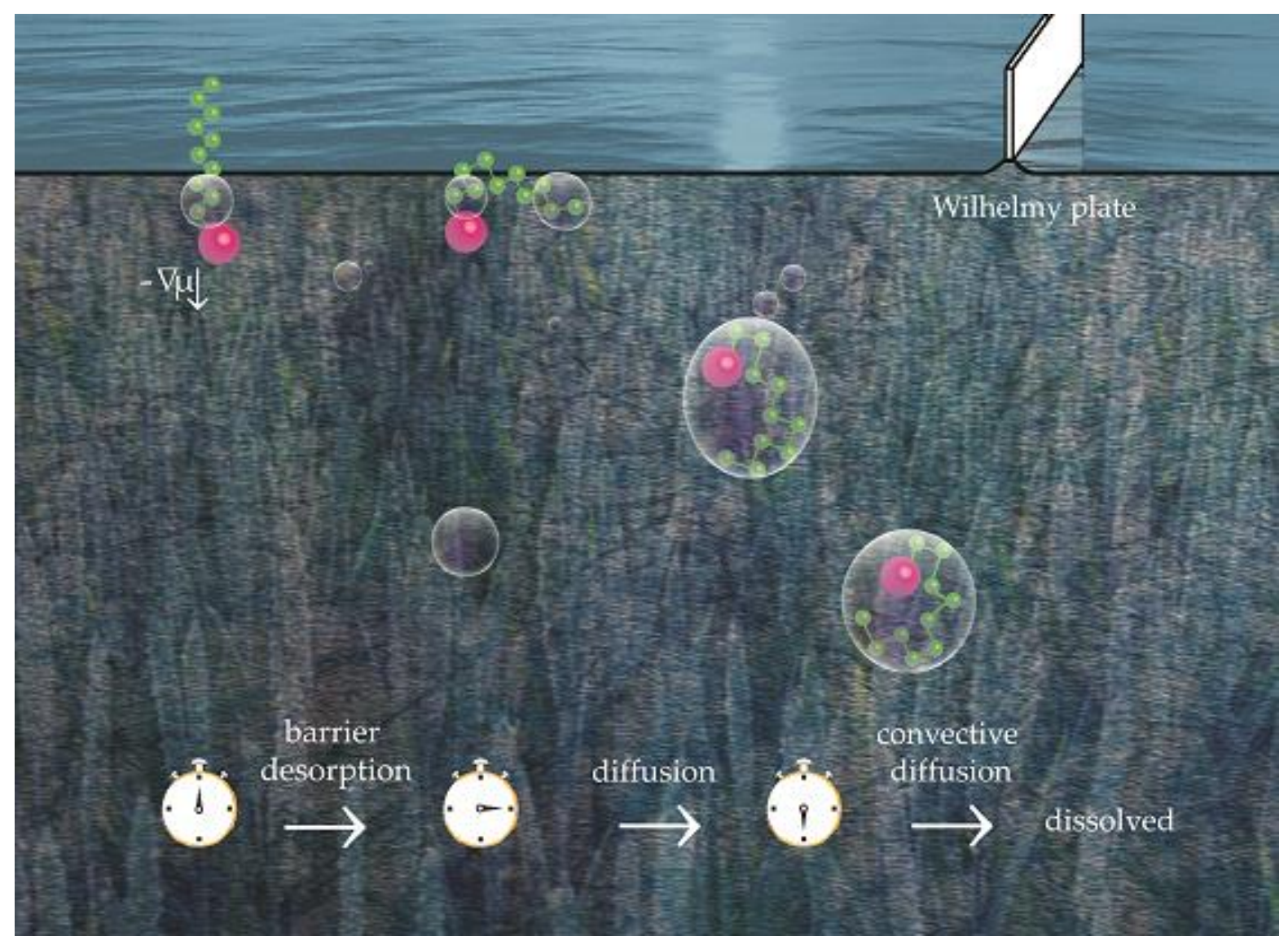

Decanol and dodecanol desorb from the surface of water following mixed barrier-diffusion kinetics. Convection accelerates the diffusion process to the point where desorption proceeds under pure barrier control, allowing accurate measurement of the rate parameters. Minkov et al. mapped the adsorption regimes as a function of convection intensity and time. 


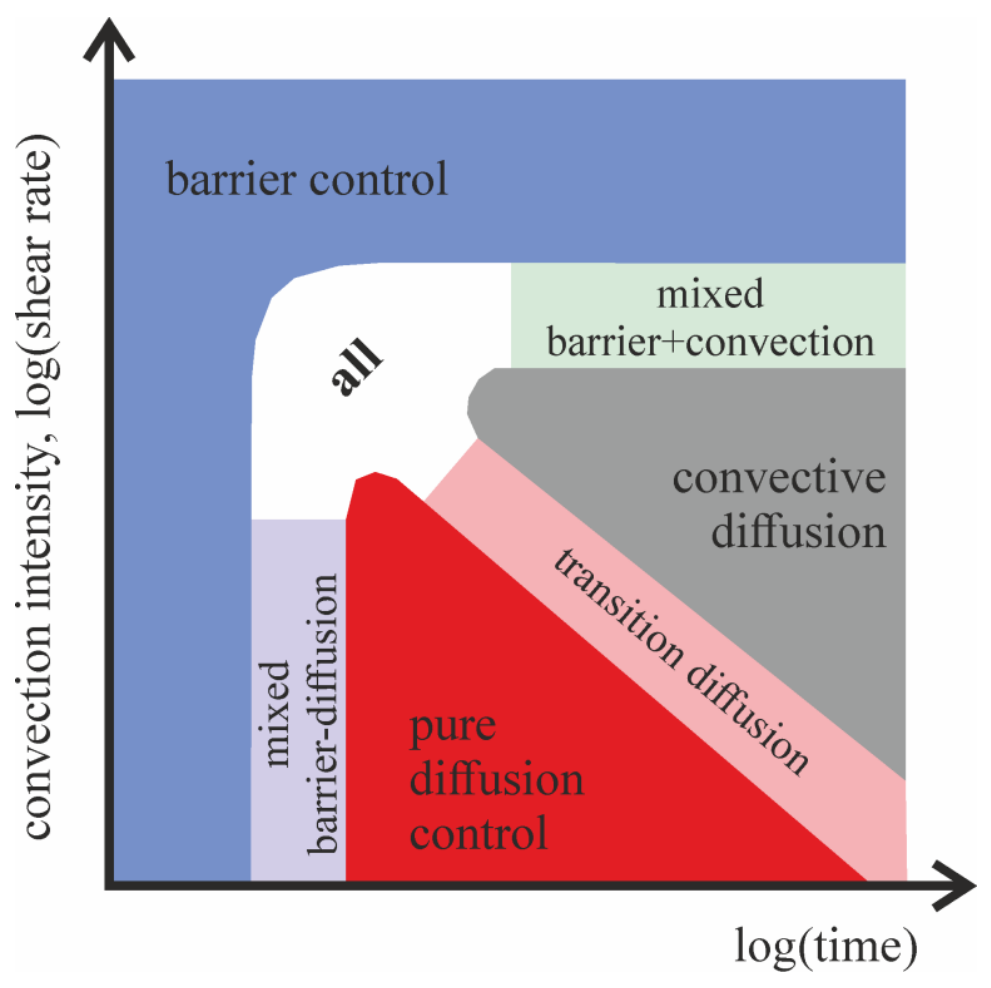

Keywords: Langmuir trough, spread monolayer, isobaric desorption, adsorption kinetics, adsorption barrier, non-ionic surfactants

\section{Introduction}

A common application [1-16] of the Langmuir trough is to follow the process of desorption of a spread monolayer of slightly soluble amphiphile under isobaric conditions, where the decrease of the area $A$ of the film with time $t$ is monitored at constant surface pressure $\pi^{\mathrm{S}}\left(\pi^{\mathrm{S}}=\sigma_{0}-\sigma\right.$, where $\sigma_{0}$ and $\sigma$ are the surface tensions of the pure solvent and of the monolayer). The isobaric regime has been used for determination of the diffusion coefficient $D$ of the surfactant [5] and for evaluation of its solubility [11]; to study the dissociation and the Hofmeister effect on the properties of monolayers of adsorbed acids [7]; to study the processes involved in the collapse of the monolayer [8,12]; phase transitions [14] in monolayers; interaction between adsorbed lipids and proteins [10]; and generally - the mechanism of desorption [4,6,9]. Recently, we used the isobaric regime as an auxiliary experiment to correct the $\pi^{\mathrm{S}}$ vs. $A$ isotherms for the material loss due to dissolution of the monolayer [17] (similarly to Motomura et al. [18]). Incidentally, we found that these auxiliary data suggested characteristics of the desorption process in apparent disagreement with certain literature results; in particular, a surprisingly slow rate of barrier desorption of dodecanol off the surface was evident. These findings were the motivation to investigate the kinetics of the desorption process in more detail.

Most isobaric studies in the field report a mechanism of the desorption process proceeding in two stages. During the first minutes after the formation of the monolayer, the 
desorption rate is controlled by a diffusion process, following approximately parabolic dependence of the area on time, $\ln \left(A_{0} / A\right) \propto t^{1 / 2}$, where $A_{0}$ is the initial area covered by the monolayer. We shall refer to this as $D$-regime, pure diffusion control, or $t^{1 / 2}$-regime. The ratedetermining process of this stage is the diffusion of surfactant through a diffusion layer of a thickness increasing with time as $(D t)^{1 / 2}$. After several minutes, another regime starts operating, where convection accelerates the diffusion and the desorption rate [3]. The approximate physical picture behind it was set by Ter Minassian-Saraga [3,4] (by analogy with the classical works on dissolution of solids [19] and diffusion-limited electrolysis [20]), and involves two layers: in the subphase next to the monolayer, a stagnated layer of liquid of thickness $L_{\mathrm{st}}$ exists, where the effect of the convection is negligible (Péclet number $P e<1$ ) and a stationary diffusion profile is established. Deeper in the bulk below the stagnated layer, another convection layer stands, where convection is assumed very efficient $(P e>>1)$, causing concentration to be equilibrated to its bulk value. We refer to this picture as $C$-regime, convective diffusion. It leads to an approximately linear dependence $\ln \left(A_{0} / A\right) \propto t$. The transition from $D$ - to C-regime occurs when $L_{\mathrm{st}} \approx(D t)^{1 / 2}$. De Keyser and Joos gave a theoretical expression for the stagnated layer thickness $L_{\mathrm{st}}$ for the case where the convection is caused by the trough's barrier movement [9]:

$$
L_{\mathrm{st}}=\left(\frac{\pi D}{2}\right)^{1 / 2}\left(\frac{\mathrm{d} \ln A}{\mathrm{~d} t}\right)^{-1 / 2} \quad\left(\sim \frac{D^{1 / 2}}{\mathrm{~d} v_{x} / \mathrm{d} z}\right) .
$$

Another common source of stirring is the natural convection, where the shear $\mathrm{d} v_{x} / \mathrm{d} z$ in eqn (1) is caused by temperature gradients. The intensity of the convection can be regulated via the thickness of the substrate [8], and by tight control of the temperature in the system. More vigorous stirring causes thinning of the stagnated layer and faster dissolution (larger $|\mathrm{d} \ln A / \mathrm{d} t|$ ). Most studies in the literature [3-5,9-16] report data that transits from $D$ - to C-regime.

A third regime has been reported to operate at short times [6]: while the diffusion layer is still thin, the rate determining process is the flip-flop-like desorption of surfactant from the monolayer to the solvent ( $\tau_{\mathrm{d}}$-regime, barrier control). This regime leads again to a linear dependence $\ln \left(A_{0} / A\right) \propto t$, but the coefficient in it is related to the energy barrier for the transfer from the monolayer to the subsurface. This behaviour is typical for very large adsorbates (proteins, nanoparticles); however, Baret et al. found a significant effect from the barrier process already for decanol [6]. De Keyser and Joos [9] plainly repudiated the work of Baret et al. as erroneous and leading to "the false conclusion that desorption is not controlled by diffusion". This was a bold statement on their side, especially in view that the first few points (first 1-2 minutes) of their own experimental $A(t)$ curves often deviate from the $\ln \left(A_{0} / A\right) \propto t^{1 / 2}$ law, approaching $\ln \left(A_{0} / A\right) \propto t$ instead, which is an indication of a barrier rate process (cf. their Fig. 1). Miller and Lunkenheimer [21] also found no need of a barrier in their interpretation of desorption data for long-chained fatty alcohols and acids, and used dynamic surface tension data to determine the diffusion coefficients for these amphiphiles, but Motomura et al. determined a barrier rate constant for myristic acid [18] at $\mathrm{pH}$ 2. In our study [17] of dodecanol 
monolayers spread on pure water or concentrated aqueous electrolytes, we found that the desorption is proceeding under barrier control for the initial 1-2 minutes. The apparent disagreements in the literature regarding the mechanism of adsorption and desorption is further discussed in the review by $\mathrm{He}$ et al. [22].

The three regimes ( $\tau_{\mathrm{d}^{-}}, D$ - and C-) do not exhaust the possibilities. Mixed barrier-diffusion ( $\tau_{\mathrm{d}} \& D-$ ) and barrier-convective diffusion ( $\tau_{\mathrm{d}} \& \mathrm{C}-$ ) control are common [6]. Other regimes have been reported, related to collapse and phase transition kinetics $[8,12,23]$ instead of solubility. The desorption rate is affected strongly by the presence of micelles [24-26]. Evaporation is an important factor [27-30] that further complicates the desorption process. Hommelen [29] found that the surface tension of alcohol monolayers is a few $\mathrm{mN} / \mathrm{m}$ lower when the surrounding air is saturated with the vapours of the alcohol solution, compared to the case where evaporation is taking place - i.e. evaporation leads to the establishment of a quasistationary alcohol adsorption lower than the equilibrium one. This effect is reversible within the first minutes of the experiment (if evaporation stops, the surface tension relaxes back to its equilibrium value), but becomes irreversible after several hours of evaporation due to decanol depletion [29]. Brooks and Alexander [27,28] reported that the increase of the salinity of the substrate does not change significantly the rate of desorption of fatty alcohols of chain length 14-18, which suggests that the loss of adsorbed material they observed is due to evaporation instead. On the other hand, the rate of desorption of $\mathrm{C}_{12} \mathrm{H}_{25} \mathrm{OH}$ decreases with the increase of the electrolyte concentration, and a significant accumulation of dissolved surfactant in the aqueous phase is observed [17,31], which proves that most of this alcohol desorbs in the liquid phase (which does not exclude evaporation).

To summarize, the barrier process of desorption is a phenomenon that is far from being well understood. Its rate has a strong dependence on $\Gamma$ that has not been studied in enough detail, and the data for it are discordant [32]. The studies of the effect of the temperature on the desorption rate are very limited (cf. ref. [24] for a brief review), and the activation energy of this process is known only to an order of magnitude. The problem is controversial - respected authors even denied the existence of a barrier process [9]. Combined with the high practical importance of the question, this makes the desorption under barrier control one of the most appealing fundamental problems in colloid science currently.

The main objectives of our study are to define the time limit between the $\tau_{\mathrm{d}}$ - and $D$-regime; to evaluate the role of the convection; to re-analyse the results of Baret at al. [6] and de Keyser and Joos [9] and to compare them with ours, hopefully reconciling them; to measure the rate of barrier desorption as a function of the monolayer compression and the temperature, and to determine the desorption energetic barrier. Another important goal is to revive the desorption isobar method - most modern adsorption-desorption kinetic studies use other techniques to follow the process (mostly methods where the dynamic surface tension is recorded as a function of time at fixed area [21,24,33-36]), and the reviews in the field from the last 20 years [24,34,37] 
hardly ever discuss this classical technique. The desorption isobars have an important advantage over the competing methods for monitoring desorption kinetics: the technique allows the process to be followed at constant $\pi^{\mathrm{S}}$ and density $\Gamma$ of the monolayer, which simplifies significantly the interpretation of the data. In contrast, a model multiparametric dependence for the desorption rate as a function of $\Gamma$ (e.g., Frumkin's, Langmuir-Hinshelwood or their derivatives [34-41]) has to be postulated in advance with most dynamic surface tension studies. We also show how the accuracy of the barrier desorption rate measurement via the isobar technique can be improved by independent measurement of the other parameters involved in the desorption process (diffusion coefficient $D$ and adsorption length $\Pi C_{\text {eq }}$ ).

The theory of the isobaric desorption under mixed barrier-diffusion regime is summarized in sec. 2. In sec. $3.1 \& 3.2$, we determine experimentally the solubility $C_{\text {eq }}$ of the dodecanol monolayer as a function of $\Gamma$, and in sec. 3.3, the diffusion coefficient $D$ is determined. The rate of desorption obtained from the desorption isobars is discussed in sec. 3.4; its dependence on $\Gamma$ and $T$ is experimentally investigated, and the activation energy for the flip-flop event is determined. We show that intensive convection can shrink the diffusion layer to negligible thickness, causing desorption under pure barrier regime. In sec. 4, our measurements are compared to literature data, and our results are summarized and presented in a schematic map of the regimes of adsorption-desorption as function of the intensity of convection and time.

\section{Desorption in the isobaric regime under barrier-diffusion control: theory}

In an isobaric desorption experiment, a surfactant monolayer is spread on the surface and then compressed quickly to a predefined value of the surface pressure $\pi^{S}$ in a Langmuir trough. The monolayer is then left to dissolve; the material lost due to dissolution is precisely compensated for by compression of the monolayer - at each time step, the electronic control system adjusts the barrier position until the predefined $\pi^{\mathrm{S}}$ is restored. Under isobaric conditions, the desorption of a single-component homogeneous adsorption layer proceeds also under constant adsorption $\Gamma$ and chemical potential of the monolayer $\mu$, as the fixed value of the $\pi^{\mathrm{S}}$ defines $\Gamma$ and $\mu$ through the equation of state of the monolayer. This assumption holds if (i) the characteristic time of the experiment is longer than the relaxation time of a monolayer of given density to its equilibrium state (fulfilled for small molecules such as dodecanol), and (ii) the bulk solution of surfactant is not concentrated (again fulfilled for dodecanol). A general discussion of the limitations of this assumption is available in ref. [42].

The driving force of the dissolution process is the difference in the chemical potential of the surfactant at the surface and in the bulk, or equivalently, between the solubility of the 
monolayer $C_{\mathrm{eq}}$ and the actual surfactant bulk concentration of the substrate (which is 0 ). The chemical potential in the monolayer is related to $\Gamma$ as

$$
\mu=\mu_{0}^{\mathrm{S}}+k_{\mathrm{B}} T \ln \gamma^{\mathrm{S}} \Gamma,
$$

where $\gamma^{\mathrm{S}}$ is surface activity coefficient, $\mu_{0}^{\mathrm{S}}$ is standard chemical potential of a surfactant molecule at the surface (a list of symbols is provided in the electronic supplement). The chemical potential $\mu$ can be calculated from the experimental surface pressure isotherm, $\pi^{\mathrm{S}}(\Gamma)$, through the integral form of the Gibbs adsorption equation:

$$
\Delta_{\mathrm{s}} \mu \equiv \mu-\mu_{\mathrm{s}}=\int_{\pi_{\mathrm{s}}^{\mathrm{s}}}^{\pi^{\mathrm{s}}} \Gamma^{-1} \mathrm{~d} \pi^{\mathrm{s}}, \quad \text { where } \quad \mu_{\mathrm{s}}=\mu_{0}^{\mathrm{s}}+k_{\mathrm{B}} T \ln \gamma_{\mathrm{s}}^{\mathrm{s}} \Gamma_{\mathrm{s}} .
$$

A graphical representation of this relation is given in Figure S17 in S4. Here, $\mu_{\mathrm{s}}, \pi_{\mathrm{s}}^{\mathrm{S}}, \Gamma_{\mathrm{s}}$ and $\gamma_{\mathrm{s}}^{\mathrm{S}}$ refer to a suitably chosen standard state. A particularly convenient standard state is that of the monolayer in equilibrium with crystals of the surfactant $[17,43]$; in this case, $\pi_{\mathrm{s}}^{\mathrm{s}}$ and $\mu_{\mathrm{s}}$ are the spreading pressure and the chemical potential of surfactant crystals, $\Gamma_{\mathrm{s}}$ is the surface density of the monolayer spread around the crystals, and $\gamma_{s}^{S}$ is its surface activity coefficient. The solubility $C_{\text {eq }}$ of the monolayer is related to its density through the condition for equilibrium between the solution (of chemical potential $\mu_{0}^{\mathrm{B}}+k_{\mathrm{B}} T \ln C_{\mathrm{eq}}$ ) and the monolayer [44,45], i.e. the adsorption isotherm

$$
\gamma^{\mathrm{S}} \Gamma=K_{\mathrm{a}} C_{\mathrm{eq}} \quad\left(\gamma_{\mathrm{s}}^{\mathrm{S}} \Gamma_{\mathrm{s}}=K_{\mathrm{a}} C_{\mathrm{s}} \text { for the standard state }\right) .
$$

Here, $K_{\mathrm{a}}=\exp \left[\left(\mu_{0}^{\mathrm{B}}-\mu_{0}^{\mathrm{S}}\right) / k_{\mathrm{B}} T\right]$ is the adsorption constant of the surfactant; for our choice of standard state, $C_{\mathrm{s}}$ is the solubility of the surfactant crystals. From eqn (2)-(4) it follows that the solubility of the monolayer $C_{\text {eq }}$ is related to the measurable quantity $\Delta_{\mathrm{s}} \mu$ as:

$$
C_{\mathrm{eq}}=C_{\mathrm{s}} \exp \left(\Delta_{\mathrm{s}} \mu / k_{\mathrm{B}} T\right) .
$$

Thus, the knowledge of $C_{\mathrm{s}}, \pi_{\mathrm{s}}^{\mathrm{S}}$, and the surface pressure vs. area isotherm allows the solubility of the monolayer at any compression to be calculated through eqn (3)\&(5) (Figure S17 in S4).

Let us now consider the change of the area of the monolayer with time. We assume that the surfactant desorbs under mixed barrier-diffusion control [6] ( $\tau_{\mathrm{d}} \& D$-regime); convection is neglected, which makes the model applicable only to the initial period of the process when $t<L_{\mathrm{st}}^{2} / D$. The evolution of the concentration profile in the bulk follows Fick's law,

$$
\frac{\partial C}{\partial t}=D \frac{\partial^{2} C}{\partial z^{2}}
$$

with initial and boundary conditions

$$
C(z=-\infty, t)=0 ; \quad C(z, t=0)=0 ;\left.\quad D \frac{\partial C}{\partial z}\right|_{z=0}=j^{\mathrm{s}} .
$$

Here, $j$ is the rate of the barrier (flip-flop) process of adsorption-desorption from the monolayer to the subsurface. For $j^{\mathrm{S}}$, we assume the following linear phenomenological relationship holds true $[6,46,47]$ :

$$
j^{\mathrm{S}}=k_{\mathrm{d}}\left[C_{\mathrm{eq}}-C(z=0)\right]=\frac{\Gamma}{\tau_{\mathrm{d}}}\left[1-\frac{C(z=0)}{C_{\mathrm{eq}}}\right] .
$$


Here, we introduced the characteristic time for desorption, $\tau_{\mathrm{d}}$ (the average time a surfactant molecule spends at the surface before desorbing), related to the desorption constant $k_{\mathrm{d}}$ as

$$
\tau_{\mathrm{d}}=\Gamma / k_{\mathrm{d}} C_{\mathrm{eq}}
$$

$\tau_{\mathrm{d}}$ is a parameter of transparent physical meaning which is going to be useful when we make comparison between different desorption kinetic models. The subsurface concentration $C(z=0)$ in eqn (8) is smaller than that of the solubility $C_{\text {eq }}$ of the monolayer because of the slow barrier processes of desorption (of rate $v_{\mathrm{d}}=k_{\mathrm{d}} C_{\mathrm{eq}}$ ) and adsorption (of rate $v_{\mathrm{a}}=k_{\mathrm{d}} C(z=0)$ ). The linear coefficient $k_{\mathrm{d}}$ and the characteristic time $\tau_{\mathrm{d}}$ are independent of the subsurface concentration, but are unknown functions of $\Gamma$ (compare to [46,35,38,39]). An immediate aim of our work is to determine experimentally $\tau_{\mathrm{d}}$ as a function of $\Gamma$.

For monolayer dissolving under conditions of constant surface tension and adsorption, the solution to the problem set by eqn (6)-(8) for the flux from the surface to the bulk reads

$$
j^{\mathrm{S}}=k_{\mathrm{d}} C_{\text {eq }} \mathrm{e}^{t / \tau_{\text {tr }}}\left(1-\operatorname{erf} \sqrt{t / \tau_{\text {tr }}}\right),
$$

where the time $\tau_{\text {tr }}$ for transition from barrier to diffusion-controlled regime [48] stands for

$$
\tau_{\mathrm{tr}}=D / k_{\mathrm{d}}^{2}=\tau_{\mathrm{d}}^{2} D C_{\mathrm{eq}}^{2} / \Gamma^{2} .
$$

In the limit of short times (thin diffusion layer, fast diffusion, barrier control), eqn (10) simplifies to a constant flux: $j^{\mathrm{S}}=k_{\mathrm{d}} C_{\text {eq }}$. In the other limit of long times (thick diffusion layer, diffusion control), a $t^{-1 / 2}$-dependence is obtained instead: $j^{\mathrm{S}}=C_{\mathrm{eq}}(D / \pi t)^{1 / 2}$. The change of the area of the monolayer under isobaric conditions follows from eqn (10) and the mass balance,

$$
\frac{1}{A} \frac{\mathrm{d} n}{\mathrm{~d} t}=\frac{\Gamma}{A} \frac{\mathrm{d} A}{\mathrm{~d} t}=-j^{\mathrm{s}},
$$

where $n=\Gamma A$ are the adsorbed moles. Upon integration of eqn (12), one obtains

$$
\ln \frac{A_{0}}{A}=\frac{\tau_{\mathrm{tr}}}{\tau_{\mathrm{d}}}\left[\mathrm{e}^{t / \tau_{\mathrm{tr}}}\left(1-\operatorname{erf} \sqrt{t / \tau_{\mathrm{tr}}}\right)-1+2 \sqrt{t / \pi \tau_{\mathrm{tr}}}\right],
$$

This result has been derived by Baret et al. [6] for the considered problem, and before that by, e.g., Munson [49], for a similar, in principle, electrochemical process. For short times, it simplifies to the linear dependence

$$
\ln \frac{A_{0}}{A}=\frac{t}{\tau_{\mathrm{d}}}
$$

Thus, at short times, the desorption is barrier controlled $[41,48]$ and the linear coefficient in the desorption law in the $\tau_{\mathrm{d}}$-regime is $1 / \tau_{\mathrm{d}}$. For long times ( $D$-regime), eqn (13) leads to

$$
\ln \frac{A_{0}}{A}=\frac{2 C_{\mathrm{eq}}}{\Gamma} \sqrt{\frac{D t}{\pi}} .
$$

However, as $t$ approaches a value of the order of $L_{\mathrm{st}}^{2} / D(\sim 100 \mathrm{~s}$ for our data below), convection accelerates the process and leads to positive deviations from eqn (13) long before its limit (15) is reached. 


\section{Determination of the rate parameters from experimental data}

The desorption kinetic equation (13) has two parameters: the average barrier desorption time $\tau_{\mathrm{d}}$ and the characteristic time $\tau_{\text {tr }}$ for transition from barrier to diffusion control, eqn (11). However, we can independently determine $\Gamma, C_{\text {eq }}$, and $D$, which leaves $\tau_{\mathrm{d}}$ as the only unknown. We will measure the first parameter, $\Gamma$, via the compression isotherm; the isotherm will be further combined with spreading pressure data to find also $\Delta_{\mathrm{s}} \mu$ (sec. 3.1). The second unknown, $C_{\mathrm{eq}}$, follows from eqn (5) using $\Delta_{\mathrm{s}} \mu$ and data for the solubility of the alcohol (sec. 3.2). The third parameter, $D$, is determined independently in sec. 3.3 using NMR data. Finally, the knowledge of $\Gamma, C_{\text {eq }}$ and $D$ allows the main desorption kinetic characteristic $-\tau_{\mathrm{d}}-$ to be accurately determined by comparing eqn (13) with desorption isobar data, sec. 3.4.

\subsection{Spreading pressure of crystals and compression isotherms $\left(\sigma_{\mathrm{s}}, \Delta \mathrm{s} \mu, \Pi\right)$}

1-dodecanol 98\% delivered by Sigma Aldrich has been used for the laboratory work. Water was double-distilled with GFL 2001/2, Germany. We took a number of measures to avoid surface active impurities compromising the results - surface rinsing, control runs and others, described in detail previously [17]. The monolayers were formed by spreading chloroform solutions of dodecanol uniformly over the available surface of water in a Teflon Langmuir trough $\left(580 \times 145 \times 4 \mathrm{~mm}^{3}\right)$. The total distance from the trough bottom to the surface (including the meniscus height) was $\sim 6 \mathrm{~mm}$. Experiments were performed at $10-30{ }^{\circ} \mathrm{C}$. The temperature of the water in the trough was controlled within $\pm 0.15^{\circ} \mathrm{C}$ by a thermostat Lauda Eco Silver RE415, Germany. The temperature of the room was set equal to that in the trough using air conditioning system; only at $10{ }^{\circ} \mathrm{C}$ in the trough, the room temperature was higher $\left(15-17{ }^{\circ} \mathrm{C}\right)$.

The surface tension was measured using a KSV Nima surface balance equipped with a platinum plate. We took measures to ensure complete wetting of the plate (pre-wetting and frequent cleaning of it, and others, described in ref. [17]). Before each experiment, we measured the surface tension of pure water, which is where surface impurities and incomplete wetting of the Wilhelmy plate affect the measurements the most. The technique reproduces the literature data with high accuracy (better than $0.05 \mathrm{mN} / \mathrm{m}$ ), as demonstrated in Figure $\mathrm{S} 11$ in $\mathrm{S} 2$; the precision, however, is lower $( \pm 0.3 \mathrm{mN} / \mathrm{m})$.

A. Spreading pressure. The equilibrium spreading tension $\sigma_{\mathrm{s}}$ of the dodecanol monolayer in contact with dodecanol crystals (or above $24.2{ }^{\circ} \mathrm{C}$, lenses) has been measured to fix the standard state of the monolayer, see eqn (3). Reproducible values are often hard to obtain; to deal with the problems involved in the measurements of $\sigma_{\mathrm{s}}$, we attempted to improve the experimental protocol we developed previously [17]. The most important steps in it are: 
(i) Preliminary saturation with $\mathrm{C}_{12} \mathrm{H}_{25} \mathrm{OH}$ of the substrate. This step is essential in order to stabilize the measurements by eliminating the monolayer desorption [17]. The saturated solutions were prepared by mixing fine dodecanol crystals with distilled water (typical treatment rate $\sim 0.1 \mathrm{~g} / \mathrm{L}$ ) and leaving the mixture to equilibrate for $12-24$ hours at the required temperature.

Using the so-obtained solution, several runs at each temperature have been performed, where each run involved:

(ii) filling the trough, and removal of the initially adsorbed material with rinsing and suction of the surface layer.

(iii) The rinsed surface of the saturated solution has been separated into two compartments by using a barrier. A small amount of fine dodecanol crystals was spread in one of the compartments, while the Wilhelmy plate was dipped into the second. The system was left in this state for $10 \mathrm{~min}$, after which the barrier was removed. This procedure was designed in order to avoid attachment of crystals to the plate - with it, the crystallites are caught by the meniscus of the trough instead of by the meniscus of the plate, and their Brownian motion is slowed down by the dense monolayer. Even when we followed this protocol, such attachments occur often. Whenever we detected an attachment, we removed, rinsed thoroughly and returned the plate into the trough.

(iv) After the barrier has been removed and the two compartments of the trough were connected, the surfaces have been left to equilibrate for 10-20 min. We then did several perturbations of the system:

- perturbation of the monolayer with the barrier through series of compressions and expansions of the monolayer of different amplitude;

- detachment and attachment of the plate;

- addition of a fresh portion of dodecanol crystals.

We previously followed a procedure that involved extrapolation using an assumed asymptotic kinetics of relaxation [17], but the acceleration of the equilibration via perturbations appears to be a better method. Each run lasted for up to 3 h. 2-4 values of the spreading pressure were recorded in each run (usually one 10-20 min after each perturbation).

The results from these measurements are presented in Figure 1 (black dots). The line is a regression over the data, and gives for the spreading tension $\sigma_{\mathrm{s}} /[\mathrm{mN} / \mathrm{m}]=28.9-0.14 \times T\left({ }^{\circ} \mathrm{C}\right)$; the standard deviation of the data off this line is $\pm 1.0 \mathrm{mN} / \mathrm{m}$. Within the precision of the measurement, the respective spreading pressure is independent of $T$ and is equal to $\pi_{\mathrm{s}}^{\mathrm{S}}=\sigma_{0}-\sigma_{\mathrm{s}}$ $=46.8 \pm 1.0 \mathrm{mN} / \mathrm{m}$. 


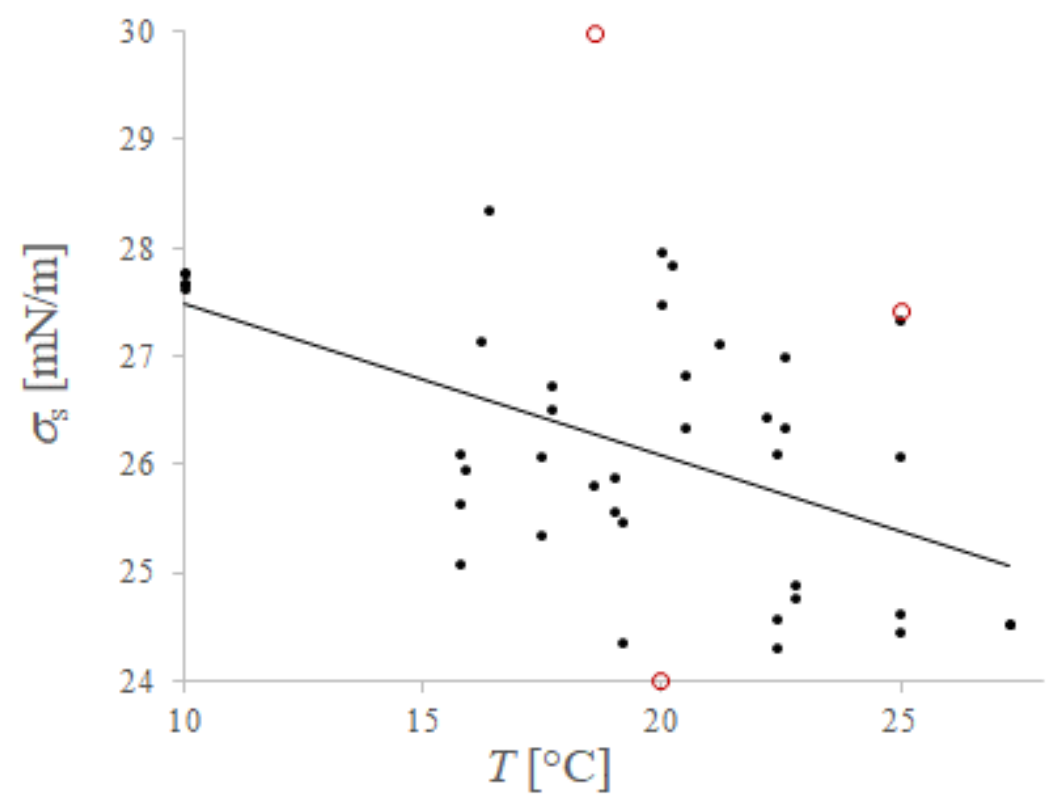

Figure 1. Spreading tension of dodecanol crystals (below $24.2^{\circ} \mathrm{C}$ ) and droplets (above $24.2{ }^{\circ} \mathrm{C}$ ) as a function of the temperature. Solid circles: Wilhelmy plate results for surface with spread crystallites.

Empty circles: bubble shape tensiometry in saturated dodecanol solution. Line: linear regression.

As a control test, we made an independent measurement of $\sigma_{\mathrm{s}}$ using profile analysis tensiometry in the emerging-bubble regime (PAT-1, Sinterface, Germany) for the same saturated solutions at 3 temperatures. At two of these temperatures, it took $4-5 \mathrm{~h}$ to saturate the surface (an example is given in Figure S12 in S3). The so-measured surface tensions of the saturated solutions are given in Figure 1 as red circles. Complete agreement with the crystal spreading pressure cannot be expected, as the crystals can alter due to diffusion of water into the crystal, i.e. the saturated solution is at chemical potential of the surfactant slightly lower from the one of the pure crystals. Attachment of crystals to the bubble is also possible. Despite these complications, the profile analysis tensiometry and the Wilhelmy plate yield values of $\sigma_{\mathrm{s}}$ in reasonable agreement with each other.

B. Compression isotherms (surface tension vs. area), and correction of the apparent area per molecule for the solubility of the monolayer. To produce the $\pi^{\mathrm{S}}(A)$ isotherms, the films were compressed by means of shifting a barrier with a constant velocity, while the change of $\pi^{S}$ with the area $A$ of the monolayer was recorded. Since we are using a sparingly soluble surfactant, the desorption process causes a steady decrease of the total adsorbed quantity $n$. The output of the apparatus is an apparent adsorption $n_{0} / A$ that is higher than the actual adsorption $\Gamma=n / A$, where $n_{0}$ is the initial quantity of surfactant spread on the substrate and $n$ is the actual adsorbed quantity after a fraction of $n_{0}$ dissolves. Therefore, the measured isotherms have to be corrected for the dissolution. To do so, we previously developed a procedure where the data for isobaric desorption are used to correct the compression experiments [17]. Here, we used a conceptually 
similar but significantly improved procedure, both more accurate and easier to perform, which is described in $\mathrm{S} 4$.

A difficult problem we experienced with the compression runs was the transport of adsorbed dodecanol through the barrier, to the free surface on the other side of the barrier. This process occurred in a random fashion: some isotherms were affected significantly, while others were not affected at all. We tried to investigate the process by placing dodecanol crystals in one compartment of the Langmuir trough, separated from a second compartment via the barrier, and measuring the surface tension in the second (Figure S20 in S5), but its random nature prevented our attempts to correct for it. The frequency of occurrence of leakages increased with the temperature and the density of the monolayer. At low temperatures $\left(<20{ }^{\circ} \mathrm{C}\right)$, one out of 2 3 isotherms was affected. Therefore, we recorded 5-6 isotherms at each $T ; 2-3$ of these were shifted to smaller areas to a random amount, and were discarded; the others agreed well and were assumed to be correct. At 23 and $25^{\circ} \mathrm{C}$, we had to do more isotherms to produce 2-3 that agree with each other. The leakages made it impossible to produce unaffected isotherms above $27^{\circ} \mathrm{C}$.

The measured isotherms are presented in Figure 2. In Figure S15 in S4, we compared them with literature results at 10 and $15{ }^{\circ} \mathrm{C}$ [50]. To facilitate the computation of $\Delta_{\mathrm{s}} \mu$ through the integral (3), we used regression formulae similarly to ref. [17], but with a simplified expression:

$$
\frac{1}{\Gamma}=\left\{\begin{array}{cc}
g_{1} \frac{1+g_{2}\left(1-\pi^{\mathrm{s}} / \sigma_{0}\right)^{m_{2}}+g_{3}\left(1-\pi^{\mathrm{s}} / \sigma_{0}\right)^{m_{3}}}{1-\left(1-\pi^{\mathrm{s}} / \sigma_{0}\right)^{m_{1}}}, & \pi^{\mathrm{s}}<\pi_{\mathrm{pt}}^{\mathrm{s}} ; \\
g_{4}-g_{5} \pi^{\mathrm{s}}, & \pi^{\mathrm{s}}>\pi_{\mathrm{pt}}^{\mathrm{s}} .
\end{array}\right.
$$

The values of the parameters $g_{i} \& m_{i}$ of this regression are tabulated in Table 4 in S4, and are compared to the experimental data in Figure 2. As in ref. [17], we corrected for the kinetics of the liquid expanded (LE)-liquid condensed (LC) phase transition (Figure S16 in S4), related to the formation of long-lived metastable structures in the heterogeneous film [51]. The measured isotherms allow the determination of the characteristics of the LE-LC phase transition (such as heat of phase transition); these are not directly relevant to the present study, but for future reference, the analysis is provided in Figure S19 in S4. 


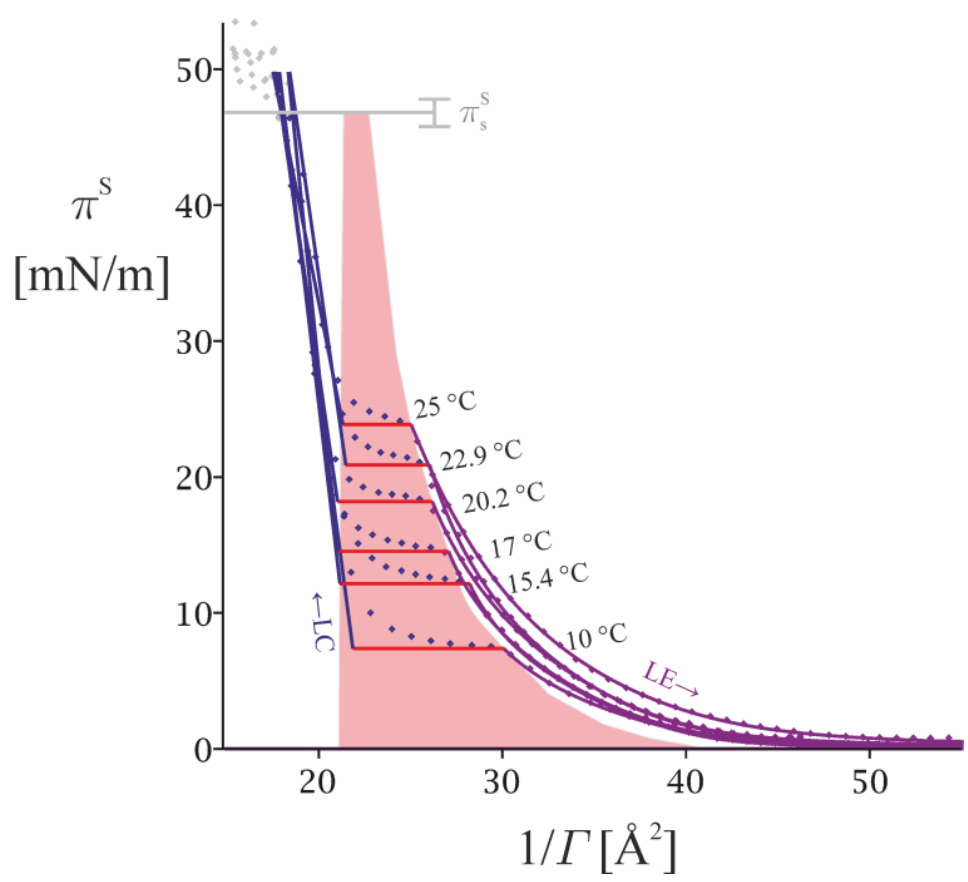

Figure 2. Surface pressure vs. area per molecule of dodecanol monolayers at various temperatures. The points are the measured $\pi^{\mathrm{s}}$; the lines are regressions with eqn (16). The grey line marks the spreading pressure of the crystals, and the respective bar represents the experimental standard deviation in its value. The shaded area under the binodal corresponds to the two-phase region of the monolayer.

\subsection{Solubility of dodecanol $\left(C_{\mathrm{s}}\right.$ and $\left.C_{\mathrm{eq}}\right)$}

The solubility of dodecanol is very low and hard to measure. The values reported in the literature vary within two orders of magnitude $\left(C_{\mathrm{s}}=0.02 \mathrm{mM}\right.$ at $25^{\circ} \mathrm{C}$ according to ref. [52,53]; $0.2 \mathrm{mM}$ from ref. [54]; $5 \mathrm{mM}$ cited in ref. [55]). In our previous work [31], we attempted to determine $C_{\mathrm{s}}$ directly from the isobaric desorption data, but this approach is inaccurate, as the effect from a larger value of $C_{\mathrm{s}}$ can be effectively compensated for by proportionally larger desorption time $\tau_{\mathrm{d}}$. Here, we use instead the solubility value $0.016 \mathrm{mM}$ at $25{ }^{\circ} \mathrm{C}$ reported by Yalkowsky and Valvani [56], which agrees well with the extrapolated value from the data of Hommelen [29].

We further used the known heats of dissolution of dodecanol to compute $C_{\mathrm{s}}$ at temperatures different from $25{ }^{\circ} \mathrm{C}$. Dodecanol is liquid at $25{ }^{\circ} \mathrm{C}$. For the heat of dissolution of the liquid $\mathrm{C}_{12} \mathrm{H}_{25} \mathrm{OH}$ in water, we used the value $h_{\mathrm{s}}^{\mathrm{L}}=10.9 \mathrm{~kJ} / \mathrm{mol}[57,58]$. The phase transition to solid dodecanol occurs at $T_{\mathrm{m}}=24.2{ }^{\circ} \mathrm{C}$. To calculate the heat $h_{\mathrm{s}}^{\mathrm{C}}$ of dissolution of the dodecanol crystals, we utilize the value of the heat of fusion of the alcohol, $h_{\mathrm{m}}=40.3 \mathrm{~kJ} / \mathrm{mol}$ at the melting temperature [59]: from it and Hess's law, we obtain $h_{\mathrm{s}}^{\mathrm{C}}=h_{\mathrm{m}}+h_{\mathrm{s}}^{\mathrm{L}}=51.2 \mathrm{~kJ} / \mathrm{mol}$. Putting these numbers together, for the solubility of dodecanol we obtain the following van't Hoff dependence: 


$$
C_{\mathrm{s}}(T)= \begin{cases}C_{\mathrm{s}, \mathrm{m}} \exp \left[-\frac{h_{\mathrm{s}}^{\mathrm{C}}}{R}\left(\frac{1}{T}-\frac{1}{T_{\mathrm{m}}}\right)\right], & T<T_{\mathrm{m}} ; \\ C_{\mathrm{s}, \mathrm{m}} \exp \left[-\frac{h_{\mathrm{s}}^{\mathrm{L}}}{R}\left(\frac{1}{T}-\frac{1}{T_{\mathrm{m}}}\right)\right], & T>T_{\mathrm{m}} .\end{cases}
$$

Here, $C_{\mathrm{m}}=0.0158 \mathrm{mM}$ is the solubility of dodecanol at the melting temperature $T_{\mathrm{m}}$. The dependence is illustrated in Figure 3. Eqn (17) involves two approximations: for negligible heat capacity of the dissolution (constant heats $h_{\mathrm{s}}^{\mathrm{C}}$ and $h_{\mathrm{s}}^{\mathrm{L}}$ in the considered temperature interval), and for negligible difference of the solubility of the pure crystals and crystals saturated with water.

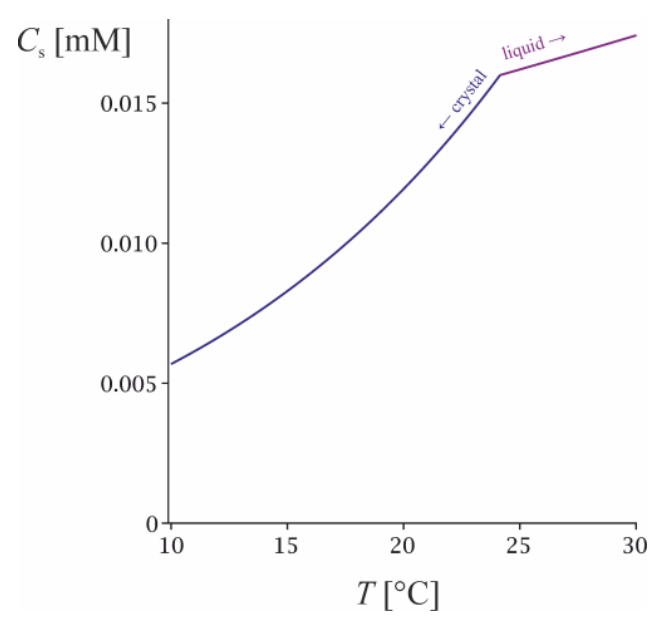

Figure 3. Solubility of $\mathrm{C}_{12} \mathrm{H}_{25} \mathrm{OH}$ in water as function of temperature, eqn (17).

In view of the disagreeing reports on the values of $C_{\mathrm{s}}$ in the literature, we designed an independent test of the value from ref. [56], as follows. The potentials $\Delta_{\mathrm{s}} \mu$ computed via eqn (3) from the compression isotherm at each surface pressure, together with eqn (5) for the solubility $C_{\text {eq }}$ of the monolayer and the value of $C_{\mathrm{s}}=0.0120 \mathrm{mM}$ at $20.2^{\circ} \mathrm{C}$ (Figure 3), allow us to deduce the surface pressure vs. concentration isotherm as done in Figure 4 (solid lines). This experimental isotherm can be compared to the sticky disc adsorption model of Ivanov et al. $[45,60,61,62]$, known to work well for the LE phase of alcohols [60]. The equation of state of a sticky disc fluid reads:

$$
\frac{\pi^{\mathrm{S}}}{k_{\mathrm{B}} T}=\frac{R_{\beta}-1}{2 \alpha \beta(1-\alpha \Gamma)}, \quad \text { where } \quad R_{\beta}=\sqrt{1+4 \beta \frac{\alpha \Gamma}{1-\alpha \Gamma}} .
$$

Here, $\alpha$ is hard-disc area of an adsorbed surfactant molecule ( $\alpha=16.5 \AA^{2}$ following from crystallographic data $[45,60])$ and $\beta$ is lateral attraction parameter (26.4 for dodecanol, theoretical value at $21^{\circ} \mathrm{C}$, cf. table 2 in the supplement of ref. [60]). The respective activity coefficient is given by [45]:

$$
\ln \gamma^{\mathrm{s}}=-\ln (1-\alpha \Gamma)+\left(2+\frac{1}{\beta}\right) \ln \frac{2}{1+R_{\beta}}+\frac{\alpha \Gamma(4-3 \alpha \Gamma)}{(1-\alpha \Gamma)^{2}} \frac{2}{1+R_{\beta}} .
$$


Together with the theoretical adsorption constant of dodecanol, $K_{\mathrm{a}}=440 \mu \mathrm{m}$ [60], this formula defines the adsorption isotherm (4) for $\mathrm{C}_{12} \mathrm{H}_{25} \mathrm{OH}$ in the LE state. The theoretical $\pi^{\mathrm{S}}$ vs. $C_{\text {eq }}$ dependence following from the sticky disc model, eqn (18),(19)\&(4), is compared with the one deduced from the experimental compression isotherms and the value of $C_{\mathrm{s}}$ in Figure 4. According to eqn (5), were the real value of $C_{\mathrm{s}}$ higher than $0.012 \mathrm{mM}$ (as suggested in ref. [52$55])$, the $\pi^{\mathrm{S}}\left(C_{\text {eq }}\right)$ curve deduced from our compression isotherms would shift proportionally to $C_{\mathrm{s}}$ on the right of the sticky disc model prediction. Instead, the agreement is excellent between 5 and $20 \mathrm{mN} / \mathrm{m}$. There is discrepancy in the low-pressure region, but this is not surprising - the sticky disc model is known to fail close to the critical point of the gaseous-LE phase transition [45], and the data for dodecanol monolayer at $\pi^{\mathrm{S}}<3-4 \mathrm{mN} / \mathrm{m}$ indeed fall into this region [45,60].

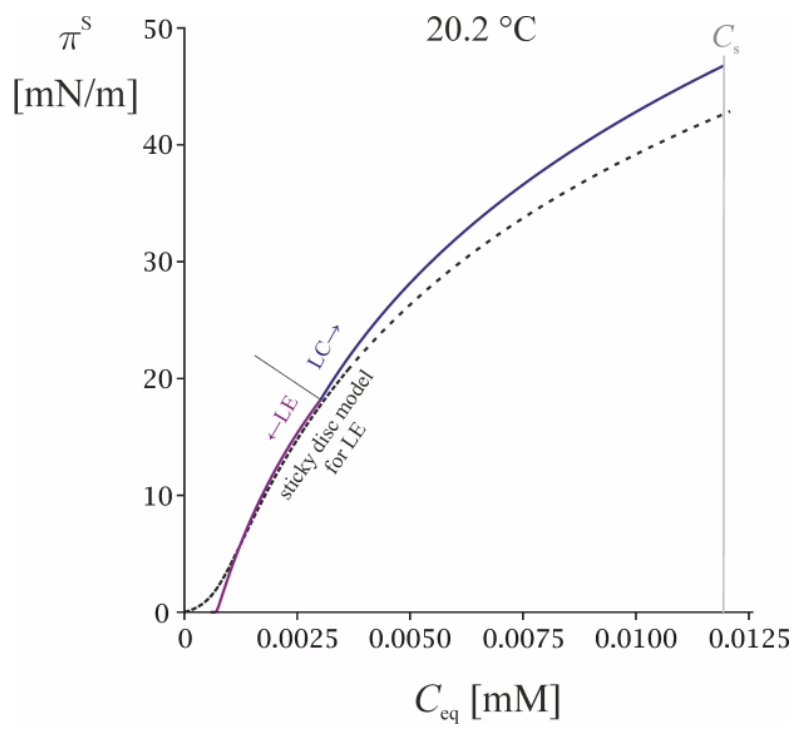

Figure 4. Equilibrium surface pressure vs. concentration isotherm. Solid lines are deduced from the compression isotherms through eqn (5)\&(3), using the value $0.0120 \mathrm{mM}$ for the solubility $C_{\mathrm{s}}$ of dodecanol crystals. The dashed line stands for the theoretical predictions from the sticky disc model, eqn (18)-(19). The agreement observed in the LE region is a confirmation that the value of $C_{\mathrm{s}}$ is correct.

\subsection{Diffusion coefficient of alcohols in water $(D)$}

Often $[39,36,63]$, when data for adsorption kinetics are interpreted, the diffusion coefficient $D$ is left as a free adjustable parameter determined from the data themselves. We made an attempt to do so ourselves, but found that our desorption kinetics data can be interpreted using various combinations of values of $D$ and $\tau_{\mathrm{d}}$ with similar success in terms of deviation of the model (13) from the data. Therefore, we instead determined $D$ independently. We are not aware of a method that would allow $D$ of $\mathrm{C}_{12} \mathrm{H}_{25} \mathrm{OH}$ in water to be directly measured, due to this alcohol's extremely low solubility. We instead measured the diffusion coefficient $D_{\mathrm{D}_{2} \mathrm{O}}$ for a few alcohols 
shorter than dodecanol in heavy water, supplemented these results with literature data, and used a simple model to extrapolate to $n=12$.

The measurements of $D_{\mathrm{D}_{2} \mathrm{O}}$ have been performed at $20^{\circ} \mathrm{C}$ using pulsed-field gradient NMR experiments. ${ }^{1} \mathrm{H}-\mathrm{NMR}$ spectra were acquired on a Bruker Avance III HD $500 \mathrm{MHz}$ spectrometer (Bruker BioSpin GmbH, Karlsruhe, Germany) with a dual ${ }^{1} \mathrm{H} /{ }^{13} \mathrm{C}$ cryoprobe. We used the standard Bruker pulse program, ledbpgp2s, employing a stimulated echo and longitudinal eddy-current delay using bipolar gradient pulses [64] with 2 spoil gradients. The diffusion coefficients $D_{\mathrm{D}_{2} \mathrm{O}}$ were calculated by fitting the experimental data with the equation [65]:

$$
I=I_{0} \exp \left[-D_{\mathrm{D}_{2} \mathrm{O}} \gamma^{2} g^{2} \delta^{2}(\Delta-\delta / 3)\right]
$$

here, $I_{0}$ is the NMR signal intensity in the absence of any gradient, $\gamma$ is the gyromagnetic ratio of ${ }^{1} \mathrm{H}$ nuclei, $g$ is the strength of the gradient pulse of duration $\delta$, and $\Delta$ is the observation time (the separation of the gradient pulses). The measurements were carried out holding constant $\delta=5 \mathrm{~ms}$ and $\Delta=100 \mathrm{~ms}$, and varying the magnetic field gradient $g$. The NMR spectra were acquired and processed using TopSpin software (Bruker $\mathrm{GmbH}$ ). Saturated aqueous solution of several n-alcohols (hexanol, octanol, nonanol and decanol, Sigma-Aldrich, >99\% pure, GC grade) in $\mathrm{D}_{2} \mathrm{O}$ were analysed. They were prepared by mixing an excess amount of the desired alcohol with $2 \mathrm{~mL}$ of $\mathrm{D}_{2} \mathrm{O}$ in a thermostated glass vials at $20{ }^{\circ} \mathrm{C}$ for $24 \mathrm{hrs}$. The undissolved alcohol was removed from the solution by filtration through a $0.22 \mu \mathrm{m}$ PTFE syringe filter.

The measured values of $\mathrm{D}_{2} \mathrm{O}$ are summarized in Table 1. The solubilities of nonanol and decanol are also too low to obtain accurate diffusion coefficients with the NMR technique, and the measured $D_{\mathrm{D}_{2} \mathrm{O}}$ for them appear to be underestimated. We attempted to measure also $D_{\mathrm{D}_{2} \mathrm{O}}$ for saturated dodecanol using the same method, but the obtained value is definitely an artefact (an order of magnitude lower than expected). The results for $D_{\mathrm{D}_{2} \mathrm{O}}$ in heavy water were reduced to $D$ in normal water using the formula

$$
D=D_{\mathrm{D}_{2} \mathrm{O}} \frac{\eta_{\mathrm{D}_{2} \mathrm{O}}}{\eta}
$$

where $\eta=1.00$ and $\eta_{\mathrm{D}_{2} \mathrm{O}}=1.25 \mathrm{mPa} \cdot \mathrm{s}$ are the respective viscosities of $\mathrm{H}_{2} \mathrm{O}$ and $\mathrm{D}_{2} \mathrm{O}$ at $20{ }^{\circ} \mathrm{C}$. To reduce the value of $D$ at $T=293 \mathrm{~K}$ to standard temperature of $T_{0}=298 \mathrm{~K}$, we used

$$
D_{0}=D(T) \frac{T_{0} \eta(T)}{T \eta_{0}}
$$

where $D_{0}$ and $\eta_{0}$ are the diffusion coefficient and the viscosity in water at $T_{0}$. Both equations are forms of the Einstein-Stokes relation, with the assumption that the Stokes radius of the considered alcohols is the same for $\mathrm{H}_{2} \mathrm{O}$ and $\mathrm{D}_{2} \mathrm{O}$, and has no significant dependence on $T$. A similar assumption has been made by others [66,24]. Stilbs [67] also used NMR to determine the diffusion coefficient of hexanol in $\mathrm{D}_{2} \mathrm{O}$ (but at $25{ }^{\circ} \mathrm{C}$, where $\eta=0.89$ and $\eta_{\mathrm{D}_{2} \mathrm{O}}=1.11$ $\mathrm{mPa} \cdot \mathrm{s})$. After correcting for the temperature dependence according to eqn (21), the result of Stilbs agrees well with ours, Figure 5. 
Table 1. Diffusion coefficient of saturated solutions of alcohols in $\mathrm{D}_{2} \mathrm{O}$ (measured) and $\mathrm{H}_{2} \mathrm{O}$ (calculated through eqn (21)\&(22)).

\begin{tabular}{|c|c|c|}
\hline & $\begin{array}{c}10^{10} \times D_{\mathrm{D}_{2} \mathrm{O}}\left[\mathrm{m}^{2} / \mathrm{s}\right] \\
\text { in } \mathrm{D}_{2} \mathrm{O}, 20^{\circ} \mathrm{C}\left(\text { measured }^{\mathrm{a}}\right)\end{array}$ & $\begin{array}{c}10^{10} \times D\left[\mathrm{~m}^{2} / \mathrm{s}\right] \\
\mathrm{H}_{2} \mathrm{O}, 25^{\circ} \mathrm{C} \text { (corrected) }\end{array}$ \\
\hline $\mathrm{C}_{6} \mathrm{H}_{13} \mathrm{OH}$ & $5.8 \pm 0.2$ & 8.0 \\
\hline $\mathrm{C}_{8} \mathrm{H}_{17} \mathrm{OH}$ & $4.48 \pm 0.015$ & 6.19 \\
\hline $\mathrm{C}_{9} \mathrm{H}_{19} \mathrm{OH}$ & $4.02 \pm 0.07$ & 5.55 \\
\hline $\mathrm{C}_{10} \mathrm{H}_{21} \mathrm{OH}$ & $3.0 \pm 0.2$ & 4.2 \\
\hline
\end{tabular}

${ }^{a}$ An average of the values obtained from the proton peaks at chemical shifts 3.681, 1.327, 1.158, 1.015 , and $0.738 \mathrm{ppm}$.

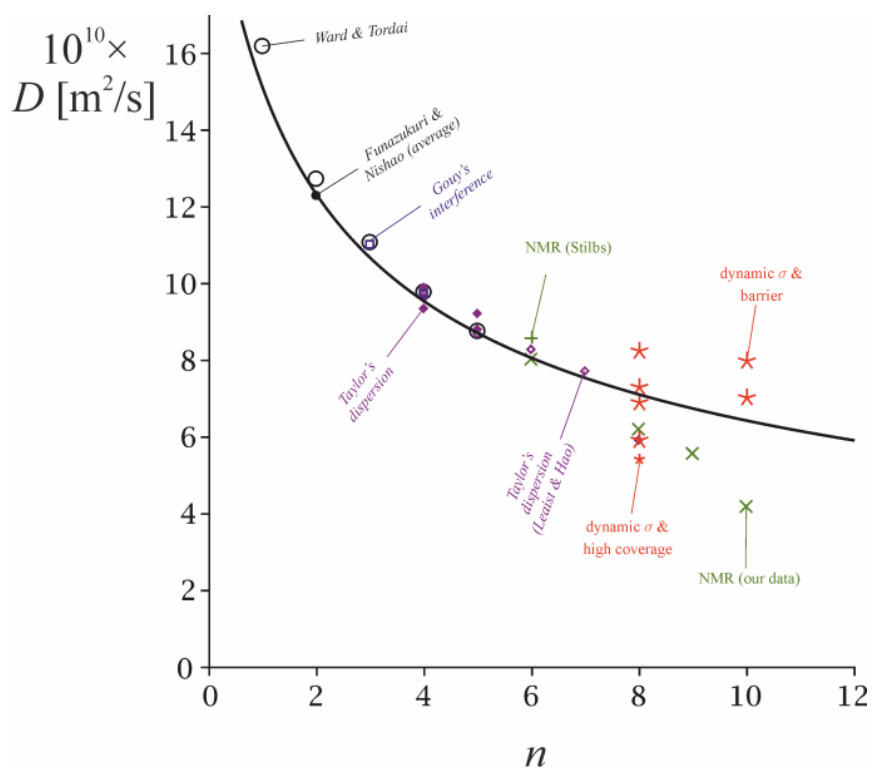

Figure 5. Diffusion coefficient of $n$-alkanols in water as a function of the number of carbon atoms in the chain. All data (ours and those from ref. $[63,66-73,76,77,39,36]$ ) are reduced to $25{ }^{\circ} \mathrm{C}$ and $\mathrm{H}_{2} \mathrm{O}$, as explained in the text. The line is the model eqn (23).

We supplemented the NMR results with $D$ values measured with Taylor's method by Leaist and Hao [68,69] and others [70-73], and by the interference method [74,75] (reducing the data to $25^{\circ} \mathrm{C}$ using eqn (22), where necessary). We utilized also the average value for ethanol from the values assembled in ref. [72], and the literature data from fig. 2 in ref. [66]. For higher alcohols, we also collected some values of $D$ that were obtained from the interpretation of dynamic surface tension data, but only when explicit account for the barrier of desorption has been taken $[76,77,39,36]$; these values are very scattered as they are sensitive to the adsorption model used by the authors, but still, they agree with the general trend of $D$ as a function of $n$ (Figure 5). There are many values of $D$ reported as extracted from dynamic surface tension measurements, but with the barrier desorption rate neglected $[63,21,78,79]$; these are nearly 
always very low compared to the general trend. The reason is that, when the flip-flop rate is ignored in an adsorption kinetic model, it can be compensated by artificial decrease of the diffusion coefficient to obtain similar dynamic surface tension curves (as noted already by Ward and Tordai [66], and analysed in detail by Liggieri et al. [80,81]). As we shall show in sec. 3.4 below, the rate of barrier desorption becomes infinite at high densities of the desorbing monolayer, and for such monolayers, the assumption for diffusion-controlled adsorption is reasonable. Unfortunately, most of the data reported in the literature refer to dilute monolayers; the only value of $D$ we found obtained in this way with relatively dense monolayer is by Defay and Hommelen [63], for $3.44 \mathrm{mM}$ octanol in water - it is also plotted in Figure 5.

To extrapolate the assembled data to dodecanol, we assumed the following scaling behaviour for $D$ with the carbon number of the alcohols:

$$
D=D_{n=0} / \sqrt{n+1} ;
$$

this is again a form of the Einstein-Stokes formula, with the assumption that the effective Stokes radius scales as $r_{\mathrm{S}}=r_{\mathrm{S}, n=0} \times(n+1)^{1 / 2}$, typical for long unbranched molecules (we use $n+1$ instead of $n$ to account for the terminal $\mathrm{OH}$ group). We determined a best fit value of the empirical parameter $D_{n=0}$ by comparing eqn (23) with the data in Figure 5 for $D$ of alcohols from ethanol to decanol; the obtained optimal value is $D_{n=0}=(21.3 \pm 1.4) \times 10^{-10} \mathrm{~m}^{2} / \mathrm{s}$. With this $D_{n=0}$, eqn (23) yields $D=(5.9 \pm 0.4) \times 10^{-10} \mathrm{~m}^{2} / \mathrm{s}$ for dodecanol at $25^{\circ} \mathrm{C}$ and $D=(6.4 \pm 0.4) \times 10^{-10}$

$\mathrm{m}^{2} / \mathrm{s}$ for decanol at $25^{\circ} \mathrm{C}$. The values of $D$ at other temperatures were calculated using again the Einstein-Stokes relation (22), in the form $D(T)=D_{0} T \eta_{0} / T_{0} \eta$. The resulting numbers are summarized in Table 2 for dodecanol and Table 3 for decanol.

\subsection{Desorption isobars of dodecanol $(\tau \mathrm{d})$}

The main body of experiments we did was desorption isobars at several surface pressures and temperatures, where the change of the monolayer area with time was recorded at constant surface pressure. We usually did 3 desorption isobars in each run on the same substrate - one at very low surface pressure, another at medium, and a last one at high. Certainly, each next isobar is affected by the previous ones (the diffusion layer already contains surfactant at the start of the $2^{\text {nd }}$ and $3^{\text {rd }}$ isobar). However, the solubility of the monolayer at the lower surface pressure is 2-3 times lower than the one at the higher, e.g., $C_{\mathrm{s}}(5 \mathrm{mN} / \mathrm{m})=0.61 \mu \mathrm{M}$ while $C_{\mathrm{s}}(20$ $\mathrm{mN} / \mathrm{m})=1.46 \mu \mathrm{M}$ at $10{ }^{\circ} \mathrm{C}$, Table 2 ; we assumed that this is enough to make the effect small. To confirm that, we repeated several high- $\pi^{S}$ isobars alone, and obtained similar rates of desorption. The final results suggest that some of the isobars at $\pi^{\mathrm{S}}>30 \mathrm{mN} / \mathrm{m}$ were affected and desorbed by $c a$. 15-20\% more slowly than expected. A related problem is that the desorption in the isobaric regime always have a prehistory (finite time for preparation of the monolayer and its compression to the prescribed $\pi^{S}$ ), which makes the determination of the initial moment and area inaccurate. Also, the control of $\pi^{\mathrm{S}}$ is imperfect: especially at low 
temperatures and high surface pressures, where the monolayers are elastic, oscillations of $\pi^{\mathrm{S}}(t)$ were observed, causing respective oscillations of $A(t)$, and in addition, often $\pi^{\mathrm{S}}$ is by $0.5-1$ $\mathrm{mN} / \mathrm{m}$ lower than the predefined value for the first 15-20 s of the isobar. The average $\pi^{\mathrm{S}}$ for the duration of the desorption isobar are reported in Table 2 (instead of the predefined one). Leakages were not frequent with the desorption isobars compared to the compression isotherms, probably due to the slower motion of the barrier; yet some have been detected, and the respective accelerated isobars were discarded.

A typical isobaric desorption curve is presented in Figure 6. It illustrates the role of the three major mechanisms of transport: barrier, pure diffusion, convective diffusion. The pure diffusion regime, eqn (15), predicts dissolution much faster than the experimentally observed (the $D$-curve in Figure 6); therefore, the observed rate of area decrease is impossible to explain without a desorption barrier - both pure diffusion, $D$-, and convective diffusion, C-, regimes overestimate the desorption rate. The pure barrier transport regime, eqn (14) and the $\tau_{\mathrm{d}}$-line in Figure 6, holds for 5-10 s (while $t<<\tau_{\mathrm{tr}}$ [48], where $\tau_{\mathrm{tr}} \approx 50 \mathrm{~s}$ from eqn (11)). The mixed barrierdiffusion regime $\tau_{\mathrm{d}} \& D$, eqn (13) and the $\tau_{\mathrm{d}} \& D$ curve in Figure 6, is in excellent agreement with the data for the first $25 \mathrm{~s}$, showing the distinctive concave shape of the $A / A_{0} \mathrm{vs}$. $t$, typical for diffusion process with diffusion layer of thickness $(D t)^{1 / 2}$ increasing with time. Between the $25^{\text {th }}$ and the $30^{\text {th }}$ second, an increase of the desorption rate is observed in comparison with the $\tau_{\mathrm{d}} \& D$ curve, as the transport through the diffusion layer passes from pure diffusion to convective diffusion regime. After the 35 second, the linear C-regime of Ter Minassian-Saraga (or a mixed $\tau_{\mathrm{d}} \& \mathrm{C}$ regime, which is also linear) is fully established.

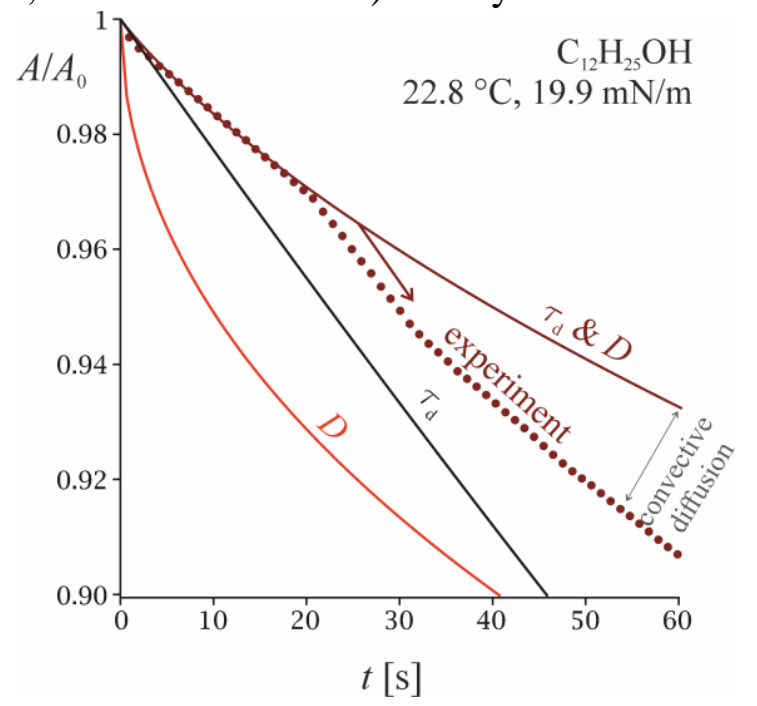

Figure 6. Desorption of dodecanol from a monolayer spread on water at $\pi^{\mathrm{S}}=19.9 \mathrm{mN} / \mathrm{m}, 22.8^{\circ} \mathrm{C}$ : relative monolayer area $A / A_{0}$ vs. time $t$. The desorption is under barrier control for the first $5 \mathrm{~s}-\tau_{\mathrm{d}}$ line, eqn (14). For $t=5-20 \mathrm{~s}$, mixed barrier-diffusion regime is established $-\tau_{\mathrm{d}} \& D$ line, eqn (13).

At $t>25 \mathrm{~s}$, convection accelerates the desorption process.

The complete suppression of convection would lead to the establishment of the $\tau_{\mathrm{d}} \& D$ regime (13). In the other limit of very intensive convection (whatever the source, trough's 
barrier movement, natural convection or stirring), the stagnation layer will become very thin, $L_{\mathrm{st}}<<\left(D \tau_{\mathrm{d}}\right)^{1 / 2}$, which will result in purely barrier-controlled desorption following eqn (14) (an example for this behaviour will be given below). Therefore, in the presence of convection, the experimental data inevitably fall between the $\tau_{\mathrm{d}}$ and the $\tau_{\mathrm{d}} \& D$ curves, as it is indeed observed in Figure 6 and all other isobars we analysed.

To extract $\tau_{\mathrm{d}}$, the desorption data have been analysed in 2 iterations, according to the following algorithm:

Iteration I. Step I-1: the point where the desorption transits from $\tau_{\mathrm{d}} \& D$ to convective diffusion $\left(\tau_{\mathrm{d}}, D \& \mathrm{C}\right)$ regime has been visually determined for each isobar. This transition often occurs with a break that is easy to identify (as in Figure 6, where it is indicated with an arrow), but sometimes it is smooth instead, and not all transition times could be accurately determined in the first iteration. Step I-2: the data falling in the $\tau_{\mathrm{d}} \& D$ regime were fitted with eqn (13), and a first-iteration values for $\tau_{\mathrm{d}}$ and $\tau_{\text {tr }}$ have been determined. These were used to correct the compression isotherms, as explained in sec. $3.1 \mathrm{~B}$.

Iteration II. Step II-1: the points where the transition from $\tau_{\mathrm{d}} \& D$ to $\tau_{\mathrm{d}}, D \& \mathrm{C}$ regime occurs were corrected wherever the parameters $\tau_{\mathrm{d}}$ and $\tau_{\mathrm{tr}}$ did not agree with the general trend. Step II-2: the values for $D$ from sec. 3.3 and for $C_{\text {eq }}$ and $\Gamma$ from sec. $3.1 \& 3.2$ were calculated (which involves the use of the corrected compression isotherms), Table 2. This leaves a single unknown in eqn (13): the average desorption time $\tau_{\mathrm{d}}$. Step II-3: all measured isobars were fitted with eqn (13) to determine $\tau_{\mathrm{d}}$. The obtained values are listed in Table 2, and Figure 7 illustrates the data at $17.1^{\circ} \mathrm{C}$, as a function of the area per molecule $1 / \Gamma$.

We usually repeated iteration II to ensure that both the $\left(\tau_{\mathrm{d}} \& D\right)$ to $\left(\tau_{\mathrm{d}}, D \& \mathrm{C}\right)$ transition point and the solubility correction of the isotherms are sufficiently accurate.

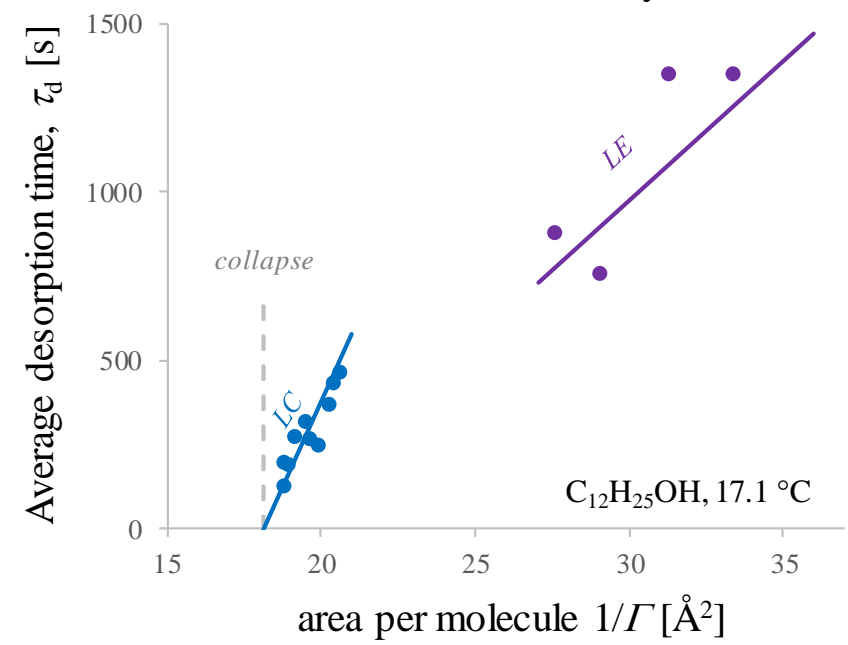

Figure 7. Average desorption time, $\tau_{\mathrm{d}}=\Pi v_{\mathrm{d}}$, vs. the area per molecule, $1 / \Gamma$, of dodecanol monolayers at $17.1^{\circ} \mathrm{C}$. Lines are eqn (24), with slopes $K^{\mathrm{LE}} \& K^{\mathrm{LC}}$ according to eqn (25) (the values of $\tau_{\mathrm{d}}, \Gamma, K^{\mathrm{LE}}$ and $K^{\mathrm{LC}}$ are those in Table 2). 
Table 2. Equilibrium and kinetic parameters for adsorption-desorption of dodecanol at various surface pressures and temperatures.

\begin{tabular}{|c|c|c|c|c|c|c|}
\hline dodecanol & $\pi^{\mathrm{S}}[\mathrm{mN} / \mathrm{m}]$ & ${ }^{\mathrm{a}} 1 / \Gamma\left[\AA^{2}\right]$ & ${ }^{\mathrm{b}} C_{\mathrm{eq}}[\mu \mathrm{M}]$ & $\mathrm{c}^{\mathrm{c}}$ fitted $\tau_{\mathrm{d}}[\mathrm{s}]$ & $\mathrm{d}^{\mathrm{d}}$ final $\tau_{\mathrm{d}}[\mathrm{s}]$ & \\
\hline \multirow{6}{*}{$\begin{array}{r}T=9.8{ }^{\circ} \mathrm{C} \\
\mathrm{e} D=3.83 \times 10^{-10} \mathrm{~m}^{2} / \mathrm{s} \\
{ }^{\mathrm{f}} C_{\mathrm{s}}=5.5 \mu \mathrm{M} \\
{ }^{\mathrm{j}} K^{\mathrm{LE}}=134 \& K^{\mathrm{LC}}=331 \mathrm{~s} / \AA^{2}{ }^{2}\end{array}$} & 5.0 & 32.4 & 0.61 & 1710 & 1940 & \multirow{2}{*}{ LE } \\
\hline & 6.8 & 30.5 & 0.71 & 1310 & 1640 & \\
\hline & 19.8 & 20.6 & 1.46 & 502 & 843 & \multirow{4}{*}{ LC } \\
\hline & 24.9 & 20.1 & 1.91 & 780 & 649 & \\
\hline & 29.6 & 19.7 & 2.48 & 412 & 500 & \\
\hline & 39.4 & 18.7 & 3.91 & 190 & 187 & \\
\hline \multirow{7}{*}{$\begin{array}{r}T=15.4{ }^{\circ} \mathrm{C} \\
{ }^{\mathrm{e}} D=4.53 \times 10^{-10} \mathrm{~m}^{2} / \mathrm{s} \\
{ }^{\mathrm{f}} C_{\mathrm{s}}=8.5 \mu \mathrm{M} \\
{ }^{\mathrm{j}} K^{\mathrm{LE}}=92 \& K^{\mathrm{LC}}=227 \mathrm{~s} / \AA^{2}\end{array}$} & 5.0 & 33.1 & 0.89 & 1650 & 1380 & \multirow{3}{*}{ LE } \\
\hline & 10.0 & 29.1 & 1.31 & 961 & 1000 & \\
\hline & 12.0 & 28.3 & 1.52 & 912 & 931 & \\
\hline & 19.9 & 20.5 & 2.31 & 906 & 526 & \multirow{4}{*}{ LC } \\
\hline & 29.9 & 19.6 & 3.80 & 675 & 327 & \\
\hline & 34.8 & 19.2 & 4.83 & 495 & 229 & \\
\hline & 39.7 & 18.7 & 6.11 & 260 & 134 & \\
\hline \multirow{12}{*}{$\begin{array}{r}T=17.1^{\circ} \mathrm{C} \\
{ }^{\mathrm{e}} D=4.76 \times 10^{-10} \mathrm{~m}^{2} / \mathrm{s} \\
{ }^{\mathrm{f}} C_{\mathrm{s}}=9.6 \mu \mathrm{M} \\
{ }^{\mathrm{j}} K^{\mathrm{LE}}=82 \& K^{\mathrm{LC}}=203 \mathrm{~s} / \AA^{2}\end{array}$} & 3.0 & 36.2 & 0.81 & 2130 & 1480 & \multirow{4}{*}{ LE } \\
\hline & 5.0 & 33.3 & 0.97 & 1355 & 1260 & \\
\hline & 7.0 & 31.2 & 1.13 & 1355 & 1070 & \\
\hline & 13.0 & 27.6 & 1.75 & 883 & 772 & \\
\hline & 20.0 & 20.6 & 2.58 & 467 & 501 & \multirow{8}{*}{ LC } \\
\hline & 22.9 & 20.4 & 3.01 & 437 & 449 & \\
\hline & 24.9 & 20.2 & 3.32 & 371 & 418 & \\
\hline & 32.9 & 19.6 & 4.95 & 271 & 295 & \\
\hline & 34.7 & 19.5 & 5.40 & 320 & 268 & \\
\hline & 39.8 & 19.1 & 6.89 & 275 & 193 & \\
\hline & 42.6 & 18.9 & 7.89 & 193 & 147 & \\
\hline & 44.6 & 18.7 & 8.64 & 134 & 117 & \\
\hline \multirow{4}{*}{$\begin{array}{r}T=19.9^{\circ} \mathrm{C} \\
\text { e } D=5.15 \times 10^{-10} \mathrm{~m}^{2} / \mathrm{s} \\
{ }^{\mathrm{f}} C_{\mathrm{s}}=11.7 \mu \mathrm{M} \\
{ }^{\mathrm{j}} K^{\mathrm{LE}}=69 \& K^{\mathrm{LC}}=169 \mathrm{~s} / \AA^{2}\end{array}$} & 4.9 & 34.5 & 1.13 & 1420 & 1130 & \multirow{2}{*}{ LE } \\
\hline & 10.0 & 29.9 & 1.68 & 761 & 806 & \\
\hline & 29.8 & 19.8 & 5.30 & 229 & 273 & $\mathrm{IC}$ \\
\hline & 34.7 & 19.2 & 6.73 & 277 & 187 & LC \\
\hline \multirow{5}{*}{$\begin{array}{r}T=22.8^{\circ} \mathrm{C} \\
{ }^{\mathrm{e}} D=5.56 \times 10^{-10} \mathrm{~m}^{2} / \mathrm{s} \\
{ }^{\mathrm{f}} C_{\mathrm{s}}=14.4 \mu \mathrm{M} \\
=57 \& K^{\mathrm{LC}}=140 \mathrm{~s} / \AA^{2}\end{array}$} & 5.0 & 34.3 & 1.40 & 942 & 927 & \multirow{3}{*}{ LE } \\
\hline & 9.9 & 29.9 & 2.06 & 383 & 673 & \\
\hline & 14.9 & 27.3 & 2.90 & 338 & 527 & \\
\hline & 24.9 & 20.1 & 5.22 & 170 & 277 & \\
\hline & 34.5 & 19.0 & 8.29 & 120 & 126 & $\mathrm{LC}$ \\
\hline \multirow{6}{*}{$\begin{array}{r}T=24.8^{\circ} \mathrm{C} \\
{ }^{\mathrm{e}} D=5.88 \times 10^{-10} \mathrm{~m}^{2} / \mathrm{s} \\
{ }^{\mathrm{f}} C_{\mathrm{s}}=16.0 \mu \mathrm{M} \\
{ }^{\mathrm{j}} K^{\mathrm{LE}}=51 \& K^{\mathrm{LC}}=124 \mathrm{~s} / \AA^{2}\end{array}$} & 4.8 & 36.4 & 1.38 & 615 & 936 & \multirow{4}{*}{ LE } \\
\hline & 6.9 & 33.8 & 1.65 & 474 & 784 & \\
\hline & 9.8 & 31.3 & 2.08 & 459 & 665 & \\
\hline & 15.0 & 28.3 & 3.01 & 259 & 522 & \\
\hline & 24.9 & 21.2 & 5.63 & 152 & 378 & \multirow{2}{*}{ LC } \\
\hline & 30.1 & 20.4 & 7.34 & 117 & 277 & \\
\hline dodecanol & $\pi^{\mathrm{S}}[\mathrm{mN} / \mathrm{m}]$ & ${ }^{\mathrm{a}} 1 / \Gamma\left[\AA^{2}\right]$ & ${ }^{\mathrm{b}} C_{\mathrm{eq}}[\mu \mathrm{M}]$ & ${ }^{\mathrm{c}}$ fitted $\tau_{\mathrm{d}}[\mathrm{s}]$ & ${ }^{\mathrm{d}}$ final $\tau_{\mathrm{d}}[\mathrm{s}]$ & \\
\hline
\end{tabular}

${ }^{\mathrm{a}}$ Determined from $\pi^{\mathrm{s}}$ through the compression isotherms in Figure 2 via the regression (16). ${ }^{\mathrm{b}}$ Obtained via eqn (3)\&(5), with $\Delta_{\mathrm{s}} \mu$ computed from the compression isotherms in Figure 2 via eqn (16) (as illustrated in Figure S17 in S4) using the spreading pressure from sec. 3.1. ${ }^{\mathrm{c}}$ Average desorption time obtained by fitting each desorption isobar with eqn (13) (1-parameter fit). ${ }^{\mathrm{d}}$ Average desorption time obtained by fitting those from column " $c$ " as function of $\Gamma$ and $T$ with the model (24)\&(25). ${ }^{\text {e }}$ Diffusion coefficient as obtained in sec. 3.3. ${ }^{\mathrm{f}}$ Solubility $C_{\mathrm{s}}$ of dodecanol crystals/droplets as obtained in sec. 3.2. ${ }^{\mathrm{j}}$ Values of the linear coefficients in eqn (24) specifying the dependence of $\tau_{\mathrm{d}}$ on $\Gamma$, as they follow from eqn (25) (with $K_{0}^{\mathrm{LE}}=50.1 \mathrm{~s} / \AA^{2}, E_{\mathrm{A}}^{\mathrm{LE}}=45.3 \mathrm{~kJ} / \mathrm{mol}$, and $K_{0}^{\mathrm{LC}}=122 \mathrm{~s} / \AA^{2}$ and $\left.E_{\mathrm{A}}^{\mathrm{LC}}=45.9 \mathrm{~kJ} / \mathrm{mol}\right)$. 
It is seen in Figure 7 that, for both the LE and LC phase, the dependence of $\tau_{\mathrm{d}}$ on the area per molecule is to a good approximation linear, as has been previously observed by us [31]. Another important feature of the dependence is that $\tau_{\mathrm{d}}$ approaches zero as the monolayer approaches the collapse point (where $1 / \Gamma$ is equal to the collapse area of the surfactant $\alpha_{\perp}$ ), i.e. the desorption rate from a collapsing monolayer is infinite. For alcohols, $\alpha_{\perp}=18.2 \AA^{2}$ (as seen in Figure 2). The linear dependence in Figure 7 can therefore be written as [31]:

$$
\tau_{\mathrm{d}}= \begin{cases}K^{\mathrm{LE}}\left(\Gamma^{-1}-\alpha_{\perp}\right) & (\mathrm{LE} \text { region }) \\ K^{\mathrm{LC}}\left(\Gamma^{-1}-\alpha_{\perp}\right) & (\mathrm{LC} \text { region }) .\end{cases}
$$

The desorption time $\tau_{\mathrm{d}}$ can be expected to have an Arrhenius dependence on the temperature. Therefore, as $\alpha_{\perp}$ is nearly independent of $T$, from eqn (24) it follows that Arrhenius dependences should also hold for the linear coefficient $K^{\mathrm{LE}}$ and $K^{\mathrm{LC}}$ :

$$
K^{\mathrm{LE}}=K_{0}^{\mathrm{LE}} \exp \left[\frac{E_{\mathrm{A}}^{\mathrm{LE}}}{R}\left(\frac{1}{T}-\frac{1}{T_{0}}\right)\right] \text { and } K^{\mathrm{LC}}=K_{0}^{\mathrm{LC}} \exp \left[\frac{E_{\mathrm{A}}^{\mathrm{LC}}}{R}\left(\frac{1}{T}-\frac{1}{T_{0}}\right)\right] .
$$

We used eqn (24)\&(25) to fit all measured $\tau_{\mathrm{d}}$ in Table 2, at all $\Gamma$ and $T$, for the LE and the LC phases separately, thus obtaining the four parameters - the standard slopes, $K_{0}^{\mathrm{LE}}=50 \pm 3 \mathrm{~s} / \AA^{2}$ and $K_{0}^{\mathrm{LC}}=122 \pm 15 \mathrm{~s} / \AA^{2}$, and the activation energies for desorption, $E_{\mathrm{A}}^{\mathrm{LE}}=45 \pm 5 \mathrm{~kJ} / \mathrm{mol}$ and $E_{\mathrm{A}}^{\mathrm{LC}}$ $=46 \pm 10 \mathrm{~kJ} / \mathrm{mol}$ (the uncertainties define the region of values of $E_{\mathrm{A}}$ and $K_{0}$ producing dispersion within $2.5 \%$ from the minimal dispersion, cf. Figure S24 in S6). The computed $\tau_{\mathrm{d}}$ are listed in the last column of Table 2, and eqn (24) is illustrated for $17.1^{\circ} \mathrm{C}$ in Figure 7.

The experimental $A(t)$ dependences at $17.1^{\circ} \mathrm{C}$ are compared in Figure 8 with the theoretical prediction (13), with $\tau_{\mathrm{d}}$ set to its "final" value following from eqn (24)\&(25), and with the theoretical transition time $\tau_{\mathrm{tr}}$ (eqn (11) with $\Gamma, C_{\text {eq }}$, and $D$ from Table 2 ). The agreement is within the uncertainty of the experimental data. The results at $10,15,20$, and $23{ }^{\circ} \mathrm{C}$ are similar and are shown in Figure S21 in S6. The acceleration of the dissolution process with the increase of $\pi^{\mathrm{S}}$ is well-represented by the model (13)\&(24), as long as the mixed $\tau_{\mathrm{d}}-D$ regime is operating. In addition, the acceleration of the desorption rate with the increase of temperature is in agreement with the Arrhenius equation (25) (cf. Table 2 and Figure S21 in S6). In most cases, the measured desorption rates at the highest surface pressures studied $(30-45 \mathrm{mN} / \mathrm{m})$ are slower than predicted by $c a .15 \%$. This is most likely due to the prehistory of the respective desorption isobars: before the "initial" moment, 1-2 other isobars at lower pressures have been measured, and the monolayer has been compressed, i.e. the substrate is of non-zero initial concentration of surfactant, which slows down the desorption.

As time advances, the experimental desorption rate becomes faster than the one predicted by eqn (13), due to transition from pure diffusion to convective diffusion transport - wherever clear, this transition is indicated with an arrow in Figure 8, and in Figure S21 in S6. The faster the decrease of $A$ with $t$, the sooner the transition to convective regime occurs, suggesting that the rate of convection correlates with the rate of desorption. This is in qualitative agreement 
with the model of De Keyser and Joos [9] - according to eqn (1), the characteristic transition time $L_{\mathrm{s} t}^{2} / D$ is proportional to $(\mathrm{d} \ln A / \mathrm{d} t)^{-1}$. However, we think it is more likely that the main convection source is the Marangoni flow produced by the surfactant diffusing below the trough's barrier to the monolayer-free compartment, a hypothesis we will test in the future. While natural convection alone cannot cause the observed effect, it is probably acting in combination with the Marangoni flow and the De Keyser-Joos convection.

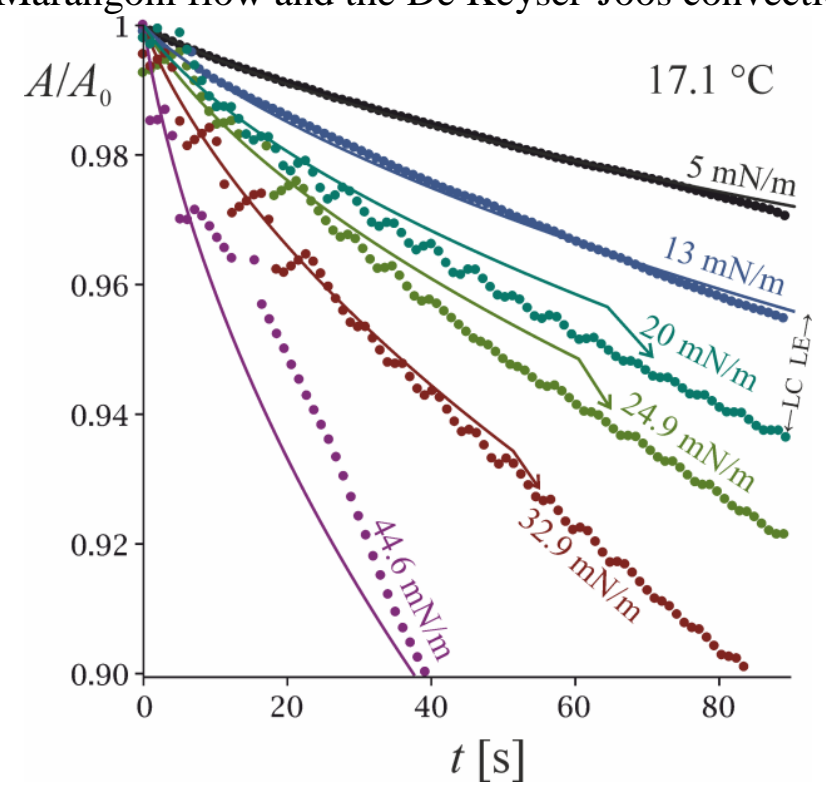

Figure 8. Desorption isobars (relative drop of the area of the monolayer vs. time) at several fixed surface pressures, $17.1^{\circ} \mathrm{C}$. At the two lowest surface pressures $(5 \& 13 \mathrm{mN} / \mathrm{m})$, the dodecanol monolayer is in the LE state, the others correspond to LC phase. Lines: the theoretical prediction (13) for mixed barrier-diffusion control, with linear dependence (24) of the desorption time $\tau_{\mathrm{d}}$ on $1 / \Gamma$ (with $K^{\mathrm{LE}}=82 \mathrm{~s} / \AA^{2}$ and $K^{\mathrm{LC}}=203 \mathrm{~s} / \AA^{2}$, Table 2). The arrows indicate transition to convective regime.

At increased temperature the transition to convective regime occurs sooner, until at $25^{\circ} \mathrm{C}$ (especially at pressure above $10 \mathrm{mN} / \mathrm{m}$ ), the convection is so intensive that the rate of convective diffusion is much faster than the rate of the barrier process. The result is that the process follows the linear regime (14) of barrier-controlled desorption. This curious case is illustrated in Figure 9: the dashed lines stand for the $\tau_{\mathrm{d}}$-regime of desorption (eqn (14) with $\tau_{\mathrm{d}}$ from eqn (24) and $K^{\mathrm{LE}}$ and $K^{\mathrm{LC}}$ from eqn (25)); the solid lines are eqn (13) of the mixed $\tau_{\mathrm{d}}-D$ regime. The experimental rate suggests that the rate-determining process is the barrier desorption. This is even more pronounced at $29-30{ }^{\circ} \mathrm{C}$, where a linear dependence of $\ln A$ against $t$ is observed for 100-200 s, in accordance to eqn (13), until most of the monolayer is dissolved (illustrated in Figure S22 in S6), with slope suggesting barrier control. 


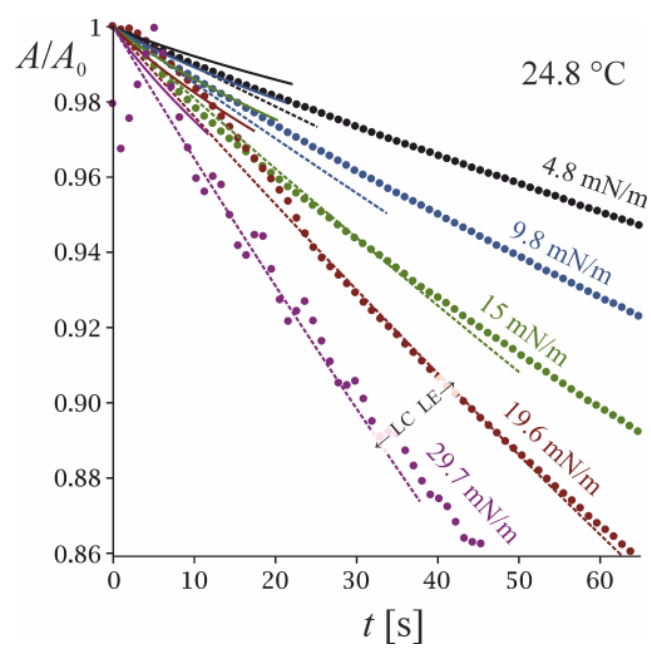

Figure 9. Desorption isobars (relative decrement of the area of the monolayer vs. time) at several fixed surface pressures and $24.8^{\circ} \mathrm{C}$. Solid lines: the theoretical prediction (13) for mixed barrier-diffusion control, with desorption time $\tau_{\mathrm{d}}$ linear with $1 / \Gamma$ (eqn (24) with $K^{\mathrm{LE}}=51 \mathrm{~s} / \AA^{2}$ and $K^{\mathrm{LC}}=124 \mathrm{~s} / \AA^{2}$, Table 2). Dashed lines: the rate of desorption under barrier control (convective diffusion much faster than the flip-flop process), according to the linear eqn (14) with the same $\tau_{\mathrm{d}}$. The data at $\pi^{\mathrm{S}}>10 \mathrm{mN} / \mathrm{m}$ agree with pure barrier regime.

\section{Discussion and conclusions}

A. Comparison with literature results. De Keyser and Joos [9] reported isobars of dodecanol at 6 and $8 \mathrm{mN} / \mathrm{m}$. Unfortunately, they did not report the temperature at which the measurements have been performed. By graphical comparison with our own data in the range $5-10 \mathrm{mN} / \mathrm{m}$, we concluded that their temperature must have been $25{ }^{\circ} \mathrm{C}$ or slightly higher. They computed the density and the solubility of the monolayer using the Langmuir-Szyszkowski adsorption model (with parameters $\Gamma_{\infty}=7 \times 10^{-6} \mathrm{~mol} / \mathrm{m}^{2}$ and $a=4.3 \times 10^{-3} \mathrm{~mol} / \mathrm{m}^{3}$ ), which is, however, inappropriate for a surfactant as strongly cohesive as dodecanol [45] - the dangers of using an unsuitable isotherm to predict kinetics has been demonstrated by Miller et al. [34]. As a result, the values of $1 / \Gamma$ computed by De Keyser and Joos are extremely high (e.g., $64.4 \AA^{2}$ at $8 \mathrm{mN} / \mathrm{m}$, instead of the correct value $32.7 \AA^{2}$ following from the isotherms in Figure 2).

De Keiser and Joos interpreted their data at long times with convective diffusion produced by the movement of the barrier and the contraction of the monolayer (with neglected barrier desorption, natural and Marangoni convection). However, in their interpretation, they used the above-mentioned erroneous values of the adsorption which are two-fold lower than the real ones. Since in their model $\mathrm{d} \ln A / \mathrm{d} t$ is proportional to $1 / \Gamma^{2}$, the actual desorption rate caused by the barrier movement is 4-fold slower than what they predicted, and the agreement they reported is fortuitous. The desorption isobar which De Keyser and Joos measured at $8 \mathrm{mN} / \mathrm{m}$ is shown in Figure 23 in S6; it refers to time range of $40 \mathrm{~min}$, with only 2-3 points falling in the range 0- 
$100 \mathrm{~s}$ which we investigate. Still, it is clear from Figure 23 that in the initial region, the data of De Keyser and Joos suggest complete barrier control, precisely as our data in Figure 9; only at longer times, the convective diffusion slows the process down. Therefore, the measurements from ref. [9] actually suggests very efficient natural or Marangoni convection (which the authors suspected) combined with a significant barrier for desorption.

Table 3. Equilibrium and kinetic parameters for adsorption-desorption of decanol at various surface pressures and temperatures.

\begin{tabular}{|r|c|c|c|c|c|}
\hline decanol & $\pi^{\mathrm{s}}[\mathrm{mN} / \mathrm{m}]$ & ${ }^{\mathrm{a}} 1 / \Gamma\left[\AA^{2}\right]$ & ${ }^{\mathrm{b}} C_{\mathrm{eq}}[\mu \mathrm{M}]$ & ${ }^{\mathrm{c}}$ fitted $\tau_{\mathrm{d}}[\mathrm{s}]$ & \\
\hline${ }^{\mathrm{d}} T=25{ }^{\circ} \mathrm{C}$ & 5.0 & 49.0 & 15.6 & 86.0 & \\
\cline { 2 - 5 }${ }^{\mathrm{e}} D=6.43 \times 10^{-10} \mathrm{~m}^{2} / \mathrm{s}$ & 10.0 & 32.6 & 25.1 & 38.1 & \multirow{2}{*}{ LE } \\
\cline { 2 - 5 }${ }^{\mathrm{f}} K^{\mathrm{LE}}=2.69 \mathrm{~s} / \AA^{2}$ & 15.0 & 27.6 & 36.1 & 20.9 & \\
\cline { 2 - 6 } & 20.0 & 25.2 & 49.9 & 14.1 & \\
\hline
\end{tabular}

${ }^{\text {a }}$ Determined from the adsorption isotherm (18) with the parameters $K_{\mathrm{a}}=36.25 \mu \mathrm{m}$ and $\beta=20.7$ obtained by comparison with surface tension vs. concentration data, Figure S25 in S7. ${ }^{\mathrm{b}}$ Obtained via eqn (4)\&(19) from the respective value of $\Gamma{ }^{\mathrm{c}}$ Average desorption time obtained by fitting each desorption isobar in Figure S26 in S7 with eqn (13) (1-parameter fit). ${ }^{\mathrm{d}}$ Baret et al. have not reported the temperature of their measurements; $25{ }^{\circ} \mathrm{C}$ is assumed by us. ${ }^{\mathrm{e}}$ Diffusion coefficient as obtained in Sec. 3.3 ${ }^{\mathrm{f}}$ Fitted value of the linear coefficient in eqn (24) specifying the dependence of $\tau_{\mathrm{d}}$ on $\Gamma$.

Let us now turn to the data of Baret et al. on decanol desorption [6]. These authors correctly interpreted their results with a mixed barrier-diffusion mechanism. However, all of the physical parameters determined by Baret et al. were listed in tables that disappeared in the published version of their manuscript (tables II- $V$ are cited but are non-existent). We therefore re-analysed their experimental curves for decanol in a manner similar to our results for dodecanol. To determine the density and the solubility of the decanol monolayers at each surface pressure reported in ref. [6], we used literature data for surface tension vs. concentration from ref. $[29,38,39,63,82]$, and interpolated them with eqn (18)-(19)\&(4), as described in S7. The results for $\Gamma$ and $C_{\text {eq }}$ are listed in Table 3 (Baret et al. did not report a temperature; we assumed $25{ }^{\circ} \mathrm{C}$, based on other works by their group). The diffusion coefficient of decanol that follows from the data in Figure 5 and eqn (23) is $D_{\text {decanol }}=6.3 \times 10^{-10} \mathrm{~m}^{2} / \mathrm{s}$ at $25^{\circ} \mathrm{C}$. This leaves the average desorption time $\tau_{\mathrm{d}}$ as the only unknown parameter in eqn (13), and we determined it for each experimental curve from ref. [6]. The comparison with the model (13) is illustrated in Figure S26 in S7, and the obtained values of $\tau_{\mathrm{d}}$ are listed in Table 3. The dependence of $\tau_{\mathrm{d}}$ on $\Gamma$ of decanol follows with reasonable accuracy the empirical relation (24) for the LE phase, with $K^{\mathrm{LE}}$ $=2.69 \pm 0.14 \mathrm{~s} / \AA^{2}$. This can be compared to $K^{\mathrm{LE}}=51 \mathrm{~s} / \AA^{2}$ for the LE phase of dodecanol at 25 ${ }^{\circ} \mathrm{C}$ (Table 2). According to eqn (24)\&(8), at a given adsorption $\Gamma$, the ratio of the desorption rates $\left(v_{\mathrm{d}}=\Pi \tau_{\mathrm{d}}\right)$ for the two alcohols is equal to the ratio of their $K^{\mathrm{LE}}$-values, as $\alpha_{\perp}$ is the same 
for decanol and dodecanol. Therefore, $\mathrm{C}_{10} \mathrm{H}_{21} \mathrm{OH}$ desorbs $51 \mathrm{~s}^{-2} / 2.69 \mathrm{~s} \AA^{-2}=19$ times faster than $\mathrm{C}_{12} \mathrm{H}_{25} \mathrm{OH}$. This means that $\tau_{\mathrm{d}}$ increases by approximately a factor of $(19)^{1 / 2}=4.35$ for each $\mathrm{CH}_{2}$ group added to the hydrocarbon chain at a given $\Gamma$ in the LE region. The result can be compared with the ratio of the barrier rates of desorption of surfactants of different chain lengths from a micelle: according to Zana et al. [83], the addition of two $\mathrm{CH}_{2}$ groups to the hydrocarbon chain of an amphiphile leads to desorption that is 10 times slower. It is noteworthy that the decanol [29] is about 17 times more soluble than dodecanol at the same temperature, confirming our previous finding [31] that the rate of barrier desorption of an amphiphile is roughly proportional to its solubility.

The order of magnitude of the values of $\tau_{\mathrm{d}}$ we obtained can be compared to the one that follows from the work of Motomura et al. [18]. Similar to other authors [35,36,37,38,39,40], they postulated that $v_{\mathrm{d}}=k_{\Gamma} \Gamma$ and $v_{\mathrm{a}}=k_{\Gamma} \Gamma_{\mathrm{eq}}$, i.e. $j^{\mathrm{S}}=k_{\Gamma}\left(\Gamma-\Gamma_{\mathrm{eq}}\right)$, where $\Gamma_{\mathrm{eq}}$ is the adsorption corresponding to monolayer in equilibrium with $C(z=0)$. Within the limits of validity of the linear approximation, this phenomenological relationship and our eqn (8) are equivalent (however, the form (8) leads to simpler final expression for $A$ vs. $t$ ). The rate parameter $k_{\Gamma}$ is related to $\tau_{\mathrm{d}}$ as $\tau_{\mathrm{d}}=\Gamma / v_{\mathrm{d}}=1 / k_{\Gamma}$. Motomura et al. found for myristic acid at $\mathrm{pH} 2$ and $25{ }^{\circ} \mathrm{C}$ an average desorption time of $\tau_{\mathrm{d}}=1 / k_{\Gamma}=3000 \mathrm{~s}$ (for $\Gamma^{-1}$ in the range 30-60 $\AA^{2}$ ), compared to $\sim 500 \mathrm{~s}$ for dodecanol. Thus, the ratio of the desorption rates of $\mathrm{C}_{13} \mathrm{H}_{27} \mathrm{COOH}$ and $\mathrm{C}_{12} \mathrm{H}_{25} \mathrm{OH}$ is of the order of 6, in agreement with what can be expected from the lengths of their hydrocarbon chains. It is also interesting to compare our $\tau_{\mathrm{d}}$ to the average time $(\sim 100 \mathrm{~s})$ for a flip-flop event of an amphiphile in a bilayer [84].

The activation energies for desorption we obtained for dodecanol, $E_{\mathrm{A}}^{\mathrm{LE}}=45 \pm 5 \mathrm{~kJ} / \mathrm{mol}$ and $E_{\mathrm{A}}^{\mathrm{LC}}=46 \pm 10 \mathrm{~kJ} / \mathrm{mol}$, can be compared with $E_{\mathrm{A}}=62 \pm 1 \mathrm{~kJ} / \mathrm{mol}$ for di(hexylglucamide), $\left(\mathrm{n}-\mathrm{C}_{6} \mathrm{H}_{13}\right)_{2} \mathrm{C}\left[\mathrm{CH}_{2} \mathrm{NHCO}(\mathrm{CHOH})_{4} \mathrm{CH}_{2} \mathrm{OH}\right]_{2}$, as estimated by Eastoe and Dalton [24], and with the estimation $\sim 40 \mathrm{~kJ} / \mathrm{mol}$ for Triton $\mathrm{X}-100$ [85,24]. Thus, the order of magnitude of this quantity also seems correct.

B. Expressions for the adsorption/desorption rates. Explicit formulae for the rate of adsorption and desorption are very useful when kinetic adsorption data for surface tension vs. time is interpreted; let us, therefore, formulate the rate expressions following from our study. Combining eqn (8),(9),(4) and the empirical eqn (24), we obtain for the LE phase the following adsorption and desorption rates:

$$
v_{\mathrm{a}}=\frac{\Gamma}{\tau_{\mathrm{d}}} \frac{C_{z=0}}{C_{\mathrm{eq}}}=\frac{K_{\mathrm{a}}}{\gamma^{\mathrm{S}}(\Gamma) K^{\mathrm{LE}}\left(\Gamma^{-1}-\alpha_{\perp}\right)} C_{z=0} \quad \text { and } \quad v_{\mathrm{d}}=\frac{\Gamma}{\tau_{\mathrm{d}}}=\frac{\Gamma}{K^{\mathrm{LE}}\left(\Gamma^{-1}-\alpha_{\perp}\right)},
$$

where $\gamma^{S}$ for short alcohols is given by eqn (19), and the adsorption constant $K_{\mathrm{a}}$ [45,60] and the kinetic parameter $K^{\mathrm{LE}}$ (eqn (25)) are well-defined functions of $T$ and the hydrocarbon chain length. For longer alcohols, $\gamma^{\mathrm{S}}$ can be instead calculated via eqn (2)-(3) from interpolated $\pi^{\mathrm{S}} \mathrm{vs.}$ $1 / \Gamma$ data, like eqn (16). In this case, the following relations can be used: 


$$
v_{\mathrm{a}}=\frac{\Gamma}{K^{\mathrm{LX}}\left(\Gamma^{-1}-\alpha_{\perp}\right)} \frac{1}{C_{\mathrm{s}} \exp \left(\Delta_{\mathrm{s}} \mu / k_{\mathrm{B}} T\right)} C_{z=0} \quad \text { and } \quad v_{\mathrm{d}}=\frac{\Gamma}{K^{\mathrm{LX}}\left(\Gamma^{-1}-\alpha_{\perp}\right)},
$$

where $K^{\mathrm{LX}}$ stands either for $K^{\mathrm{LC}}$ or $K^{\mathrm{LE}}$, depending on the surface phase in question.

The linear dependence (24) of the average desorption time $\tau_{\mathrm{d}}$ on the area per molecule $1 / \Gamma$ (Figure 7) can be expected to hold only within the LE and the LC regions of monolayers adsorbed at the water|air surface. Eqn (26)\&(27), respectively, are not suitable for monolayers sparser than those we investigated $\left(1 / \Gamma<40 \AA^{2}\right)$. For dilute gaseous monolayer, $\tau_{\mathrm{d}}$ should instead approach a constant value $\tau_{\mathrm{d}}^{\mathrm{G}}$ (independent of $\Gamma$ ) that is characteristic of a single amphiphile molecule at the neat surface (compare to the situation in, e.g., ref. [86]). Consequently, another kinetic rate formula should hold true for the 2D gas:

$$
v_{\mathrm{a}}=\frac{\Gamma}{\tau_{\mathrm{d}}^{\mathrm{G}}} \frac{C_{z=0}}{C_{\mathrm{eq}}}=\frac{K_{\mathrm{a}}}{\tau_{\mathrm{d}}^{\mathrm{G}}} C_{z=0} \quad \text { and } \quad v_{\mathrm{d}}=\frac{\Gamma}{\tau_{\mathrm{d}}^{\mathrm{G}}},
$$

where we used that $\Gamma=K_{\mathrm{a}} C_{\text {eq }}$ for infinitely dilute monolayers. Eqs (26)-(28), together with the experimental or theoretical equations of state, (16)\&(3) or (19), and the rules formulated above for the dependence on $T$ and the chain length of $\tau_{\mathrm{d}}$, define completely the phase-specific barrier adsorption-desorption rate of alcohol monolayers.

Monolayers with a structure different from that of an uncharged LE, LC or gaseous layer at water|air cannot be a priori assumed to follow the same adsorption-desorption rate laws (26) -(28). The cases of non-cohesive uncharged monolayers, charged monolayers, monolayers at solid surfaces etc. have to be studied separately.

The rate laws (24),(26)\&(27) can be compared to the popular generalized Frumkin model [35-40]; its kinetic parameters $a$ and $E_{\mathrm{d}}$ are related to the average adsorption time as $\tau_{\mathrm{d}}=a^{-1} \exp \left(E_{\mathrm{d}} / R T\right)$, where $E_{\mathrm{d}}=$ constant $+v \Gamma^{\mathrm{n}}$ (many authors [87-89,32] used a variant with $\mathrm{n}=1$, i.e. $E_{\mathrm{d}}=$ constant $+v \Gamma$ ). The value of the constant $v$ is supposed to be positive; however, for $v>0$, the dependence of $\tau_{\mathrm{d}}$ on $\Gamma$ corresponds to rate of desorption decreasing as the monolayer is compressed. On the contrary, our data suggests that as the collapse point is approached, the desorption rate increases to infinity. For many alcohols, negative values of $v$ have been reported (e.g., table 3 of ref. [32]), which produces a trend in agreement with our observations. In any case, all popular barrier kinetic models in the literature postulate a dependence of $\tau_{\mathrm{d}}$ (or equivalently, of the desorption rate $v_{\mathrm{d}}$ ) on $\Gamma$ that is rather arbitrary. The approach we use instead determines a single experimental $\tau_{\mathrm{d}}$ at each fixed value of $\Gamma$, and is, therefore, more straightforward.

C. Map of desorption regimes. A succinct summary of the regime transitions we observed experimentally can be made by plotting them onto a map of the desorption regimes as function of time and convection intensity. The map is constructed schematically based on the theoretical results from ref. [6,9,3,4,41,48] and is shown in Figure 10. As discussed in ref. [41,48], at vanishingly small times $\left(t<\tau_{\mathrm{tr}}\right.$, see eqn (11)), the desorption is always under barrier control. 
If the convection is negligible, at $t \sim \tau_{\mathrm{tr}}$, the mixed $\tau_{\mathrm{d}}-D$ regime is acting instead, and pure diffusion control ( $D$-regime) holds at $t \gg \tau_{\mathrm{tr}}$, Figure 10. However, as the time advances, the diffusion layer becomes thicker and, once $t \sim L_{\mathrm{st}}^{2} / D$, it reaches the thickness of the stagnated layer [3,4]. The location of the stagnated layer (where $P e \sim 1$ ) corresponds to a boundary between pure diffusion region near the surface and convective diffusion region far from it. From eqn (1) and the condition $L_{\mathrm{st}} \sim(D t)^{1 / 2}$, we find that the shear rate and the time for transition to convective regime are related as $\mathrm{d} v_{x} / \mathrm{d} z \sim 1 / t$. Therefore, nearby the line $\mathrm{d} v_{x} / \mathrm{d} z \sim 1 / t$, the desorption process will proceed under transitional diffusion regime $(D \& \mathrm{C}$ region in Figure 10). In our experiments, this transition from pure diffusion to convective diffusion regime often occurred with a characteristic jump (the arrows in Figure 6 and Figure 8). Finally, at high enough intensity of the convection, the boundary between convective diffusion control and pure barrier control is reached, where $\tau_{\mathrm{d}} \sim L_{\mathrm{st}}^{2} / D$. Using that $L_{\mathrm{st}}^{2} \sim D /\left(\mathrm{d} v_{x} / \mathrm{d} z\right)$, this simplifies to $\mathrm{d} v_{x} / \mathrm{d} z \sim 1 / \tau_{\mathrm{d}}-$ this condition corresponds to the horizontal zone of mixed barrier-convective diffusion control $\left(\tau_{\mathrm{d}} \& \mathrm{C}\right)$. At even higher shear rates, pure barrier regime is once again established. In the zone nearby the point where $\mathrm{d} v_{x} / \mathrm{d} z \sim 1 / \tau_{\mathrm{d}}$ and $t \sim \tau_{\mathrm{tr}}$ simultaneously, all three factors ( $\tau_{\mathrm{d}}, D \& \mathrm{C}$ ) contribute significantly. This schematic regime map is of general validity, independent of the source of shear, the type of the monolayer or interface.

A desorption isobar can be represented as a continuous curve on this map. For example, the succession of regime transitions observed in Figure $6\left(\tau_{\mathrm{d}} \rightarrow \tau_{\mathrm{d}}, D \rightarrow \tau_{\mathrm{d}}, \mathrm{C}\right)$ corresponds to the lower arrow in Figure 10. All isobars observed at $T \leq 20^{\circ} \mathrm{C}$ followed a similar pattern. On the other hand, all isobars we measured at $T>25{ }^{\circ} \mathrm{C}$ (Figure 9, Figure S22 in S6) follow the pattern illustrated by the higher arrow in Figure 10 . At $20-25^{\circ} \mathrm{C}$, the type of isobar depends on the surface pressure: the high-pressure ones are under barrier regime, while the low-pressure isobars are transient.

The regime transition temperature range we observed is close to the melting temperature $T_{\mathrm{m}}$ of dodecanol. Therefore, we investigated whether a correlation exists between the two, i.e. if more "liquidy" monolayers would desorb under barrier regime. However, the answer is negative. First, the transition range $\left(20-25{ }^{\circ} \mathrm{C}\right.$ for our data) must shift to lower temperatures at higher shear - according to our map, if the convection is intensive enough, all isobars will eventually proceed under barrier control irrespective of $T$ and the fluidity of the monolayer. In addition, the decanol melts at $6.4{ }^{\circ} \mathrm{C}$, while the regime transition temperature in the experiments of Baret et al. is above $25{ }^{\circ} \mathrm{C}$. Therefore, the proximity between $T_{\mathrm{m}}$ and the transition temperature range for dodecanol is a fortuitous coincidence. 


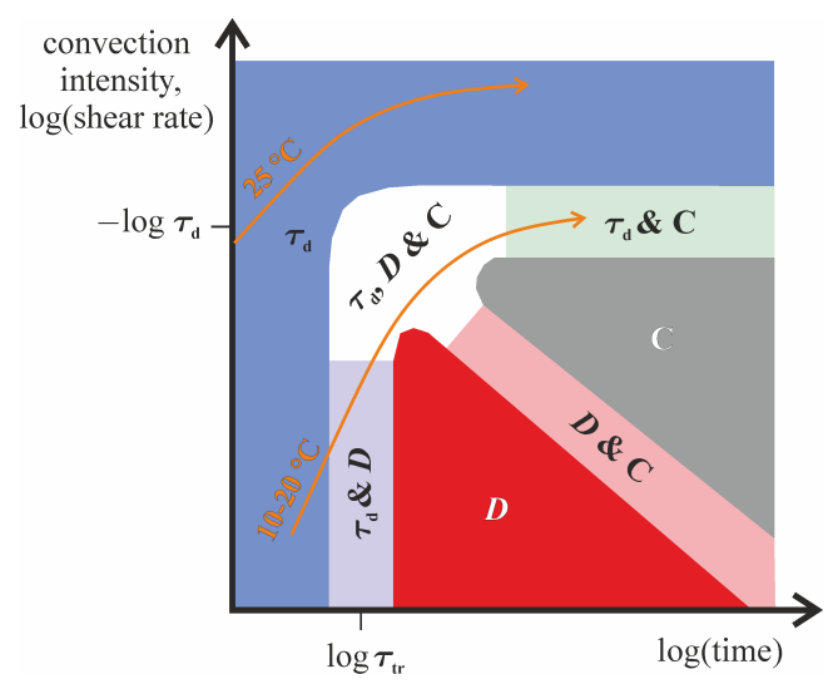

Figure 10. Map of the regimes of desorption from a flat surface against time after spreading the monolayer and convection intensity.

D. Conclusion. Let us finally summarize the most important findings of our study of the mechanism of desorption of alcohols from monolayers spread on water|air.

(i) The first important conclusion from our dodecanol data in Figure 6, Figure 8, Figure $\mathrm{S} 26$ in S7 etc. is that a significant slowdown of the desorption process occurs due to an existing adsorption/desorption barrier. It could be argued that the same data can be fitted with pure diffusion or mixed diffusion-convection mechanism if the diffusion coefficient is left as a fitting parameter. However, the resulting value of $D$ will be dubious - by a factor of about 2 smaller than the expected one $\left(4-6 \times 10^{-10} \mathrm{~m}^{2} / \mathrm{s}\right.$ for dodecanol at $10-25{ }^{\circ} \mathrm{C}$, sec. 3.3). Evaporation and convection cannot explain the slowdown - they can only accelerate the process compared to the pure diffusion-controlled desorption. The only plausible explanation for the decrease of the desorption rate compared to pure diffusion is, therefore, a slow rate of barrier desorption. Note, however, that the obtained values of $\tau_{\mathrm{d}}$ are correct only if there is no significant contribution from evaporation and convection; it is actually possible that the values we report set only a lower limit for the real ones, i.e. it is possible that the desorption barrier process is even slower but the decrease of the area is accelerated by the simultaneous processes of convection and evaporation.

(ii) We used a combination of four experiments to determine accurately the rate of barrier desorption - the auxiliary compression isotherms, spreading pressure, and NMR measurements are used to determine $\Gamma, C_{\mathrm{eq}}$ and $D$, and then the desorption isobars are used for $\tau_{\mathrm{d}}$ alone.

The rate of barrier desorption was experimentally analysed as a function of $\Gamma$, surfactant structure and temperature. The data show that the characteristic time for desorption $\left(\tau_{\mathrm{d}}=\right.$ $\Pi$ desorption rate) decreases with the compression of the monolayer, approaching zero (infinite desorption rate) near the monolayer collapse, cf. eqn (24) and Figure 7. This allows us to give a curious kinetic definition of "collapse point" as the conditions under which the rate of flip- 
flop desorption becomes infinite (thus giving a possible answer of question \#1 from the list of Franses et al., "what are the causes and events which trigger monolayer collapse?" [90]). The dependence of $\tau_{\mathrm{d}}$ on the area per molecule $1 / \Gamma$ is approximately linear, eqn (24). The dependence of the rate on temperature for dodecanol follows the Arrhenius form (25) with activation energy of 45-46 kJ/mol. The addition of each $\mathrm{CH}_{2}$ group leads to decrease of the barrier desorption rate by a factor of approximately 4.35 . The order of magnitude of these values agrees with the scarce data available in the literature.

These results can be used to test different hypotheses about the molecular mechanism of the flip-flop process behind the barrier desorption process. Two particularly interesting hints are the following features of the process:

- the activation barrier is approximately independent of the density of the monolayer in the $\mathrm{LE}$ and $\mathrm{LC}$ regions $\left(E_{\mathrm{A}}^{\mathrm{LE}}=45.3 \pm 5\right.$ and $\left.E_{\mathrm{A}}^{\mathrm{LC}}=45.9 \pm 10 \mathrm{~kJ} / \mathrm{mol}\right)$;

- the rate of barrier desorption increases quickly with the length of the hydrocarbon tail, suggesting that the energy barrier rises roughly by $4 \mathrm{~kJ} / \mathrm{mol}(1.5 \times R T)$ per $\mathrm{CH}_{2}$.

Shin and Abbott [86] hypothesized tentatively that the molecular origin of the barrier is related to the dynamics of hydrophobic solvation, which seems to agree with these two features. Yet, the question remains open.

(iii) We gave experimental evidence for pure barrier control of the desorption process at high convection rates, Figure 9.

(iv) As stated in the introduction, one of our main aims in this work is to revive the desorption isobar method to study the kinetics of adsorption-desorption. The method has an important advantage over the competing techniques: the desorption is followed at constant monolayer density. The data is relatively easy to collect and simple to interpret. The method has, of course, some disadvantages. With respect to its precision, the first source of error is the low precision in the values of $C_{\mathrm{s}}$ (probably $\left.\pm 10 \%\right), \pi_{\mathrm{s}}^{\mathrm{S}}( \pm 1 \mathrm{mN} / \mathrm{m})$, and $D( \pm 7 \%)$. The second is the systematic errors in the isobars due to the prehistory of the experiment. The technique can be optimized with respect to both these issues - e.g., a correction for the prehistory can be done similarly to the correction we did on the compression isotherms, S4. A limitation of the method is that only sparingly soluble monolayers can be studied with it, which restricts its applicability to surfactants of solubility of the order $10^{-4}-10^{-1} \mathrm{mM}$, in a limited temperature and surface pressure range. Yet the level of detail the method yields is unachievable with any other technique we are aware of, which more than compensates for these disadvantages.

\section{Acknowledgements}

The discussions with Dr. Javor Novev, Oxford University, helped us with the interpretation of the data falling in the convective diffusion regime. The work is funded by National Science Fund through Contract № 80-10-131 from 25.04.2018 with Sofia University. 


\section{Notes and references}

Electronic supplementary information available: (S1) List of symbols. (S2) The Wilhelmy method and the surface tension of water. (S3) Measuring the spreading tension with bubble profile analysis in saturated aqueous dodecanol. (S4) Correcting the adsorption isotherms for the solubility of the dodecanol and the kinetics of the LE-LC phase transition. (S5) Leakage through the barrier. (S6) Dodecanol - additional data. (S7) Decanol data.

1. J. T. Davies and E. Rideal. Interfacial phenomena. Academic Press, 1963. Chapter 4.

2. A. W. Adamson and A. P. Gast. Physical chemistry of surfaces, $6^{\text {th }}$ ed. Wiley, 1997. Sec. IV-7.

3. L. Ter Minassian-Saraga, J. Chim. Phys., 1955, 52, 181-200.

4. L. Ter Minassian-Saraga, J. Colloid Sci., 1956, 11, 398-418.

5. G. S. Patil, R. H. Matthews and D. G. Cornwell, J. Lipid Research, 1973, 14, 26-31.

6. J. F. Baret, A. G. Bois, L. Casalta, J. J. Dupin, J. L. Firpo, J. Gonella, J. P. Melinon and J. L. Rodeau, J. Colloid Interface Sci., 1975, 53, 50-60.

7. G. S. Patil, R. H. Matthews and D. G. Cornwell. Estimation of ionization in unstable fatty acid monolayers from desorption kinetics. Relationships between ionization, field strength, and cation selectivity. Advances in Chemistry, 1975, vol. 144. Chap. 5.

8. R. D. Smith and J. C. Berg, J. Colloid Interface Sci., 1980, 74, 273-286.

9. P. De Keyser and P. Joos, J. Colloid Interface Sci., 1983, 91, 131-137.

10. F. MacRitchie, J. Colloid Interface Sci., 1985, 105, 119-123.

11. D. J. Chaiko and K. Osseo-Asare, Solvent Extraction Ion Exchange, 1987, 5, 287-299.

12. D. Vollhardt and U. Retter, J. Phys. Chem., 1991, 95, 3723-3727.

13. P. Joos and M. van Uffelen, J. Colloid Interface Sci., 1993, 155, 271-282.

14. J. P. Slotte and S. Illman, Langmuir, 1996, 12, 5664-5668.

15. N. V. Romeu, P. Dynarowicz-Łątka, J. M. Trillo, J. R. Seoane and O. C. Mouzo, J. Colloid Interface Sci., 1998, 198, 179-182.

16. D. J. Chaiko and K. Osseo-Asare, Colloid Surfaces A, 2000, 175, 335-347.

17. T. V. Peshkova, I. L. Minkov, R. Tsekov and R. I. Slavchov, Langmuir, 2016, 32, 88588871.

18. K. Motomura, A. Shibata, M. Nakamura and R. Matuura, J. Colloid Interface Sci., 1969, $29,623-628$.

19. A. A. Noyes and W. R. Whitney, Z. physical. Chem., 1897, 23, 689-692.

20. W. Nernst, Z. physical. Chem., 1904, 47, 52-55.

21. R. Miller and K. Lunkenheimer, Colloid Polymer Sci., 1986, 264, 357-361.

22. Y. He, P. Yazhgur, A. Salonen and D. Langevin, Adv. Colloid Interface Sci., 2015, 222, 377-384. 
23. A. Casandra, B. A. Noskov, M.-Y. Hu and S.-Y. Lin, J. Colloid Interface Sci. 2018, 527, 49-56.

24. J. Eastoe and J. S. Dalton, Adv. Colloid Interface Sci. 2000, 85, 103-144.

25. C. D. Dushkin, I. B. Ivanov and P. A. Kralchevsky, Colloids Surf. 1991, 60, 235-261.

26. K. D. Danov, D. S. Valkovska and P. A. Kralchevsky, J. Colloid Interface Sci., 2002, $251,18-25$.

27. J. H. Brooks and A. E. Alexander, Proceedings of the Third International Congress of Surface Activity (Cologne), 1960, 4, 196-201.

28. J. H. Brooks and A. E. Alexander, J. Phys. Chem., 1962, 66, 1851-1853.

29. J. R. Hommelen, J. Colloid Science, 1959, 14, 385-400.

30. R. S. Hansen, J. Phys. Chem., 1960, 64, 637-664

31. R. I. Slavchov, I. L. Minkov, D. Arabadzieva and E. Mileva. Barrier desorption from sparingly soluble alkanol monolayers on water under constant surface tension. Nanoscience \& Nanotechnology: Nanostructured materials application and innovation transfer, 2018, issue 18.

32. C.-H. Chang and E. I. Franses, Colloid Surf., 1995, 100, 1-45.

33. S. Dukhin, G. Kretzschmar and R. Miller. Dynamics of adsorption at liquid interfaces. Theory, experiment, application. Elsevier, 1995, Amsterdam.

34. R. Miller, E. V. Aksenenko and V. B. Fainerman, Adv. Colloid Interface Sci., 2017, 247, 115-129.

35. S.-Y. Lin, K. McKeigue and C. Maldarelli, AIChE J., 1990, 36, 1785-1795.

36. D. O. Johnson and K. J. Stebe, J. Colloid Interface Sci., 1996, 182, 526-538.

37. R. Miller, A. V. Makievski and V. B. Fainerman. In: V. B. Fainerman, D. Möbius and R. Miller, eds. Surfactants - chemistry, interfacial properties, applications. Elsevier, 2001, Chapter 4.

38. S.-Y. Lin, K. McKeigue and C. Maldarelli, Langmuir, 1991, 7, 1055-1066.

39. S.-Y. Lin, T.-L. Lu and W.-B. Hwang, Langmuir, 1995, 11, 555-562.

40. P. N. Pan, J. Green and C. Maldarelli, J. Colloid Interface Sci., 1998, 205, 213-230.

41. T. D. Gurkov, Colloid Polym. Sci., 2011, 289, 1905-1915.

42. A. I. Rusanov and V.A. Prokhorov. Interfacial tensiometry. Elsevier, 1996. Sec. 14.

43. A. Frumkin and A. Pankratov, Acta Physicochim. URSS 1939, 10, 55-64.

44. R. I. Slavchov, I. M. Dimitrova and I. B. Ivanov. Cohesive and non-cohesive adsorption of surfactants at liquid interfaces. In: R. G. Rubio, Y. S. Ryazantsev, V. M. Starov, G. X. Huang, A. P. Chetverikov, P. Arena, A. A. Nepomnyashchy, A. Ferrús and E. G. Morozov, eds. Without bounds: a scientific canvas of nonlinearity and complex dynamics. Springer-Verlag, 2013.

45. R. I. Slavchov and I. B. Ivanov, Soft matter, 2017, 13, 8829-8848.

46. R. P. Borwankar and D. T. Wasan, Chem. Eng. Sci., 1983, 38, 1637-1649. 
47. Z. Adamczyk, J. Colloid Interface Sci. 1987, 120, 477-485.

48. S. N. Moorkanikkara and D. Blankschtein, J. Colloid Interface Sci., 2006, 296, 442457.

49. R. A. Munson, J. Phys. Chem., 1962, 66, 727-729.

50. V. B. Fainerman, R. Miller, E. V. Aksenenko and A. V. Makievski. In: V. B. Fainerman, D. Möbius and R. Miller, eds. Surfactants - chemistry, interfacial properties, applications. Elsevier, 2001, Chapter 3.

51. H. M. McConnell, Ann. Rev. Phys. Chem. 1991, 42, 171-195.

52. ICSC database, www.ilo.org/dyn/icsc, October 2017.

53. C. C. Addison and S. K. Hutchinson, J. Chem. Soc. 1949, 3387-3395.

54. C. L. Yaws, J. R. Hopper, S. D. Sheth, M. Han and R. W. Pike, Waste Management, 1997, 17, 541-547.

55. Chemical Book, http://www.chemicalbook.com, October 2017.

56. S. H. Yalkowsky, S. C. Valvani, J. Pharm. Sci., 1980, 69, 912-922.

57. L. Benjamin, J. Phys. Chem., 1964, 68, 3575-3581.

58. M. H. Abraham, J. Chem. Soc., Faraday Trans. 1, 1984, 80, 153-181.

59. J. C. van Miltenburg, G. J. K. van den Berg and M. Ramirez, J. Chem. Eng. Data, 2003, $48,36-43$.

60. R. I. Slavchov and I. B. Ivanov, J. Colloid Interface Sci. 2018, 532, 746-757.

61. T. D. Gurkov and I. B. Ivanov. In Proc. 4th World Congress on Emulsions, Lyon, 2006, p. 509.

62. I. B. Ivanov, K. D. Danov, D. Dimitrova, M. Boyanov, K.P. Ananthapadmanabhan and A. Lips, Colloids Surfaces A, 2010, 354, 118-133.

63. R. Defay and J. R. Hommelen, J. Colloid Sci., 1959, 14, 411-418.

64. D. H. Wu, A. D. Chen and C. S. Johnson, J. Magnetic Resonance A 1995, 115, 260264.

65. E. O. Stejskal and J. E. Tanner, J. Chem. Phys. 1965, 42, 288-292.

66. A. F. H. Ward, L. Tordai, J. Chem. Phys. 1946, 14, 453-461.

67. P. Stilbs, J. Colloid Interface Sci. 1982, 87, 385-394.

68. D. G. Leaist, J. Solution Chem., 1991, 20, 175-186.

69. L. Hao and D. G. Leaist, J. Chem. Eng. Data, 1996, 41, 210-213.

70. T. Tominaga and S. Matsumoto, J. Chem. Eng. Data, 1990, 35, 45-47.

71. T. Tominaga, S. Yamamoto and J.-I. Takanaka, J. Chem. Soc., Faraday Trans. 1, 1984, 80, 941-947.

72. T. Funazukuri and M. Nishio, J. Chem. Eng. Data 1999, 44, 73-76.

73. S. F. Y. Li and H. M. Ong, J. Chem. Eng. Data, 1990, 35, 136-137.

74. S. Rehfeldt and J. Stichlmair, Fluid Phase Equilibria, 2010, 290, 1-14.

75. P. A. Lyons and C. L. Sandquist, J. Am. Chem. Soc., 1953, 75, 3896-3899. 
76. K. Kinoshita, E. Parra and D. Needham, J. Colloid Interface Sci., 2017, 488, 166-179.

77. S.-Y. Lin, W.-J. Wang and C.-T. Hsu, Langmuir 1997, 13, 6211-6218.

78. G. Bleys and P. Joos, J. Phys. Chem. 1985, 89, 1027-1032.

79. C. M. Phan, Can. J. Chem. Eng. 2010, 88, 688-692.

80. F. Ravera, L. Liggieri and A. Steinchen, J. Colloid Interface Sci. 1993, 156, 109-116.

81. L. Liggieri, F. Ravera and A. Passerone, Colloid Surf. A 1996, 114, 351-359.

82. S.-Y. Lin, Y.-C. Lee, M.-W. Yang and H.-S. Liu, Langmuir, 2003, 19, 3164-3171.

83. J. Lang and R. Zana. Chemical relaxation methods. In: R. Zana R, ed. Surfactant Solutions. Marcel Dekker, 1987, New York, 405-452.

84. R. I. Slavchov, T. Nomura, B. Martinac, M. Sokabe and F. Sachs, J. Phys. Chem. B 2014, 118, 12660-12672.

85. R. Miller, V. B. Fainerman, K.-H. Schano, A. Hofmann and W. Heyer, Tenside Sur. Det., 1997, 34, 357-363.

86. J.Y. Shin and N.L. Abbott, Langmuir, 2001, 17, 8434-8443.

87. V. B. Fainerman, Colloid J. USSR, 1977, 39, 91-96.

88. C.-H. Chang and E.I. Franses, Colloids Surf., 1992, 69, 189-201.

89. C. A. MacLeod and C. J. Radke, J. Colloid Interface Sci., 1994, 166, 73-88.

90. E. I. Franses, C.-H. Chang, J. B. Chung, K. C. McGinnis, S. Y. Park, and D. J. Ahn. Dynamic adsorption and tension of spread or adsorbed monolayers at the air-water interface. In: D. O. Shah, ed. Micelles, microemulsions and monolayers. Marcel Dekker, 1998. Ch. 18, sec. vii. 


\title{
Barrier kinetics of adsorption-desorption of alcohol monolayers on water under constant surface tension (supplementary information)
}

Ivan L. Minkov, ${ }^{\mathrm{a}, \mathrm{b}}$ Dimitrinka Arabadzhieva, ${ }^{\mathrm{c}}$ Ibrahim E. Salama, ${ }^{\mathrm{d}, \mathrm{e}}$ Elena Mileva, ${ }^{\mathrm{c}}$ Radomir I. Slavchov*,f,g

\begin{abstract}
a Department of Physical Chemistry, Faculty of Chemistry and Pharmacy, Sofia University, 1 J. Bourchier Blvd., 1164 Sofia, Bulgaria

${ }^{\mathrm{b}}$ Department of Chemistry, Biochemistry, Physiology, and Pathophysiology, Faculty of Medicine, Sofia University, 1 Koziak Str., 1407 Sofia, Bulgaria

${ }^{\mathrm{c}}$ Bulgarian Academy of Science, Akad. G. Bonchev Str., bl.11, 1113 Sofia, Bulgaria, Sofia, Bulgaria

${ }^{\mathrm{d}}$ Department of Chemisty, Cambridge University, CB2 1EW Cambridge, United Kingdom

e BP Institute, Cambridge University, Bullard Laboratories, Madingley Road, CB3 OEZ Cambridge, United Kingdom

${ }^{\mathrm{f}}$ Department of Chemical Engineering and Biotechnology, Cambridge University, Philippa Fawcett Drive, West Site, CB3 OAS Cambridge, United Kingdom

${ }^{\mathrm{g}}$ School of Engineering and Materials Science, Queen Mary University of London, Mile End Road, London E1 4NS, United Kingdom

*E-mail: ris26@cam.ac.uk
\end{abstract}

\section{S1. List of symbols}

$A \quad$ area covered by the monolayer

$A_{0} \quad$ area covered by the monolayer in the initial moment

$C$ concentration of the surfactant

$C(z=0) \quad$ subsurface surfactant concentration (right next to the surface)

$C_{\text {eq }} \quad$ equilibrium surfactant concentration with respect to monolayer, $C_{\text {eq }}=\gamma^{\mathrm{S}} \Gamma / K_{\mathrm{a}}$

$C_{\mathrm{s}} \quad$ solubility of surfactant crystals

$D \quad$ diffusion coefficient of the surfactant

$j^{\mathrm{S}} \quad$ rate of the (monolayer) $\rightarrow$ (subsurface) barrier process of desorption, $j^{\mathrm{S}}=v_{\mathrm{d}}-v_{\mathrm{a}}$

$K^{\mathrm{LC}} \quad$ the empirical coefficient in eqn (24) for $\tau_{\mathrm{d}} \mathrm{vs} . \Gamma^{-1}$ in the LC phase

$K^{\mathrm{LE}} \quad$ the empirical coefficient in eqn (24) for $\tau_{\mathrm{d}} \mathrm{vs} . \Gamma^{-1}$ in the LE phase

$K_{\mathrm{a}} \quad$ adsorption constant of the surfactant

$k_{\mathrm{B}} \quad$ Boltzmann constant

$k_{\mathrm{d}} \quad$ rate constant for desorption, $v_{\mathrm{d}}=k_{\mathrm{d}} C_{\mathrm{eq}}$

$k_{\Gamma} \quad$ rate constant for desorption of Motomura et al., $v_{\mathrm{d}}=k_{\Gamma} \Gamma$

$n \quad$ amount of surfactant in the monolayer [mol] 


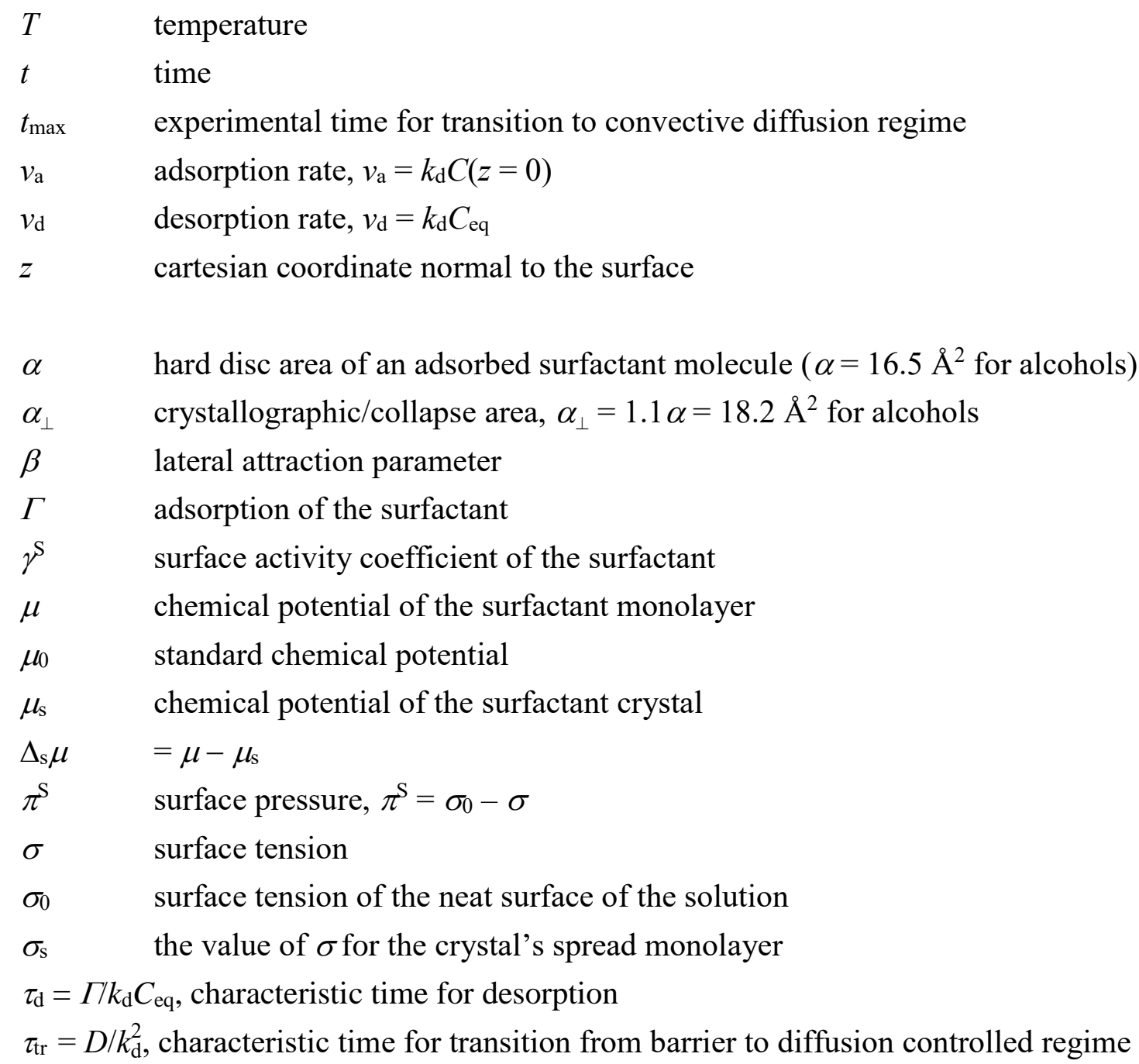

\section{S2. The Wilhelmy method and the surface tension of water}

To determine the surface tension, we measured the weight of a $19.53 \mathrm{~mm}$ wide platinum plate attached at the studied surface. As all techniques for surface tension measurement, the combined Langmuir trough/Wilhelmy plate method is not straightforward to use and requires special measures to be taken against artefacts. The main problems associated with it are

(i) fouling with surface active impurities has to be avoided;

(ii) complete wetting of the plate is required;

(iii) the location of the plate with respect to the surface has to be fixed (slow evaporation can lead to a decrease of the water level, and fast barrier movement can increase the water level, affecting the results);

(iv) a number of dynamic effects (kinetics of adsorption and desorption; natural convection and convection due to the movement of the barrier; leakage of surfactant through the barrier; evaporation of the water, the surfactant, and the organic solvent in which the surfactant is 
dissolved upon spreading; deboarding, collapse and slow kinetics of phase transitions in the monolayer) have to be considered when the data is interpreted;

(v) in the case where the spreading pressure of crystals is measured, crystals often attach to the plate and alter the signal.

Details on the procedures that we use against these artefacts as a standard in our laboratory are given in ref. [17] (surface rinsing, cleaning and prewetting of the plate etc.). With regard to (i) and (ii), pure water is the worst-case scenario: in the presence of a dense monolayer of the studied surfactant, impurities affect little the state of the surface, and the wetting is significantly improved. Therefore, we measured the surface tension $\sigma_{0}$ of pure water before each experiment. The measured $\sigma_{0}$ are shown in Figure $\mathrm{S} 11$ as a function of $T$. The line is a linear regression over the data, and gives $\sigma_{0} /[\mathrm{mN} / \mathrm{m}]=75.64-0.138 T\left({ }^{\circ} \mathrm{C}\right)$, in excellent agreement with the accepted literature values. However, the standard deviation is significant: $0.3 \mathrm{mN} / \mathrm{m}$.

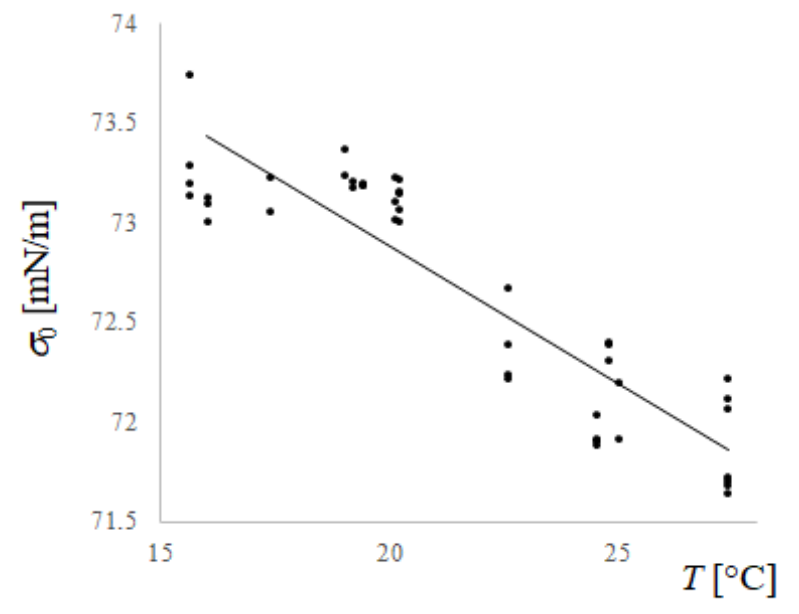

Figure S11. Surface tension of water.

\section{S3. Measuring the spreading tension with bubble profile analysis in saturated aqueous dodecanol}

For the measurements with bubble profile analysis tensiometer we used the same saturated dodecanol solutions as for experiments with the Langmuir trough. The work area of the apparatus is set to the required temperature by a thermostat. A quartz crystal cuvette is filled with $25 \mathrm{~mL}$ solution, saturated at the desired temperature. The cuvette is then covered with a top through which a glass capillary is fitted and dipped into the solution. An air bubble with a fixed area is formed at the tip of the capillary end and then the experiment is started. The apparatus is recording the surface tension variation with time, caused by dodecanol adsorption. During the experiment the surface area of the bubble is kept constant. The duration of the experiments was 4-6 hours, and continued until the surface tension vs. $t$ curve reaches a plateau value. 


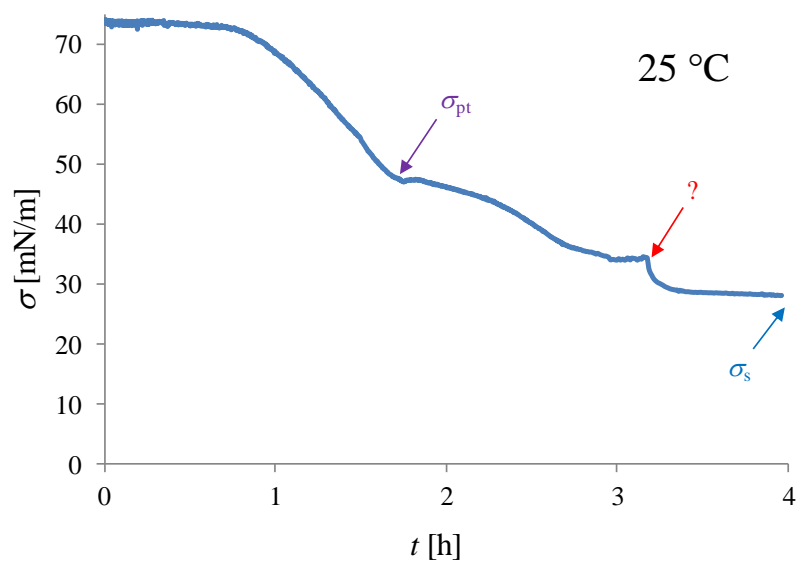

Figure S12. Dynamic surface tension of a bubble in saturated $\mathrm{C}_{12} \mathrm{H}_{25} \mathrm{OH}$ solution at $25{ }^{\circ} \mathrm{C}$.

The first kink in Figure S12 (the small plateau portion indicated with $\sigma_{\mathrm{pt}}$ ) corresponds to the LE-LC phase transition. We are unsure what could be the reason the second kink (marked with “?”) - we observed the same feature at $17{ }^{\circ} \mathrm{C}$. No similar feature is observed at this particular surface tension in the $\sigma$ vs. $1 / \Gamma$ isotherm, see Figure 2. The shape of it suggests a sudden increase in the rate of the adsorption, which might correspond to a convection transition similar to those occurring in the isobaric desorption experiments (the arrow in Figure 6). The curve reaches a plateau after $4 \mathrm{~h}$, corresponding to saturation of the surface and to value of $\sigma$ equal to the spreading tension of crystals $\left(\sigma_{\mathrm{s}}\right.$ in the Figure $\left.\mathrm{S} 12\right)$.

\section{S4. Correcting the adsorption isotherms for the solubility of the dodecanol and the kinetics of the LE-LC phase transition}

We compressed the monolayer as quickly as possible to minimize the losses due to dissolution (a compression run takes less than a minute). This option has a price - the monolayer needs some time to relax to its equilibrium state, especially in the region where the phase transitions gas-LE and LE-LC occur. If the compression is fast enough, we can approximately assume that the desorption is under barrier control. In this case, the flux of surfactant out of the surface is given by $j^{\mathrm{S}}=k_{\mathrm{d}} C_{\mathrm{eq}}=\Gamma / \tau_{\mathrm{d}}$ and the mass balance of the surface is given by the equation

$$
\frac{1}{A} \frac{\mathrm{d} n}{\mathrm{~d} t}=-\frac{\Gamma}{\tau_{\mathrm{d}}},
$$

compare to eqn (12). This equation is valid for both the isobaric runs and the normal compressions. For the isobaric regime of the Langmuir balance, where the adsorption $\Gamma$ is constant, eqn (1) leads to

$$
\frac{1}{A} \frac{\mathrm{d} A}{\mathrm{~d} t}=-\frac{1}{\tau_{\mathrm{d}}}, \text { and } \ln \frac{A}{A_{0}}=-\frac{t}{\tau_{\mathrm{d}}}
$$

i.e. eqn (14). 


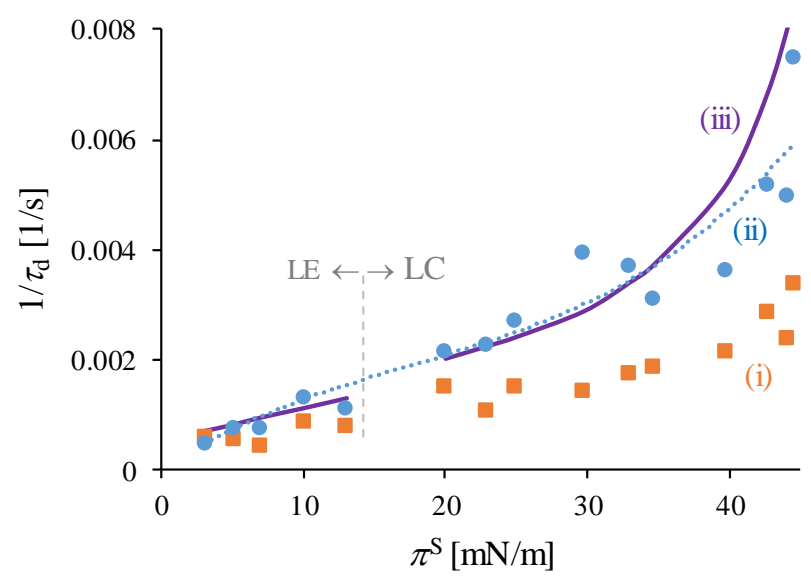

Figure S13. Reciprocal desorption time $1 / \tau_{\mathrm{d}}$ as a function of the surface pressure: results from isobaric desorption experiments at $17{ }^{\circ} \mathrm{C}$. Orange squares: $1^{\text {st }}$ iteration (2-parametric fits with eqn (13)). Blue circles: $2^{\text {nd }}$ iteration (1-parametric fits with eqn (13)). Blue dot line: polynomial regression with eqn (3). Solid line: last iteration, eqn (24)\&(16).

For every temperature and every surface pressure value, we fitted the isobaric data with eqn (13), as a first iteration, in order to determine both $\tau_{\mathrm{d}}$ and $\tau_{\mathrm{tr}}$. The results are not very accurate, but still good enough for the correction. The data for $1 / \tau_{\mathrm{d}}$ as function of $\pi^{\mathrm{S}}$ are given in Figure S13 (orange squares). The results were interpolated using the regression formula:

$$
\frac{1}{\tau_{\mathrm{d}}}=c_{1} \pi^{\mathrm{S}}+c_{2}\left(\pi^{\mathrm{S}}\right)^{2}+c_{3}\left(\pi^{\mathrm{S}}\right)^{3}
$$

We found that a quadratic or cubic polynomial is sufficient for the regression. The desorption time $\tau_{\mathrm{d}}$ is assumed to tend to infinity when $\pi^{\mathrm{S}}=0$ (infinitely dilute monolayer); therefore, eqn (3) has no constant term.

With $\tau_{\mathrm{d}}$ known, we can proceed to the integration of the kinetic equation (1) for the normal compression run to obtain the dependence of the total adsorbed quantity on time, $n(t)$. We multiply both sides of eqn (1) by $\Gamma^{-1} \mathrm{~d} t$, and use that $n / A=\Gamma$ :

$$
\frac{\mathrm{d} n}{n}=-\frac{\mathrm{d} t}{\tau_{\mathrm{d}}}
$$

Integration yields:

$$
\ln \frac{n}{n_{0}}=-J, \quad \text { where } J=\int_{0}^{t} \tau_{\mathrm{d}}^{-1} \mathrm{~d} t
$$

The integral $J$ can be computed at each time step of the compression run using the following recurrent formulation of the Newton trapezium method:

$$
J_{1}=t_{1} \tau_{\mathrm{d}, 1}^{-1} / 2 ; \quad J_{i+1}=J_{i}+\left(t_{i+1}-t_{i}\right)\left(\tau_{\mathrm{d}, i+1}^{-1}+\tau_{\mathrm{d}, i}^{-1}\right) / 2 ;
$$

here, $\tau_{\mathrm{d}, i}=\tau_{\mathrm{d}}\left(\pi_{i}^{\mathrm{S}}\right)$, as given by the interpolation (3), and $\pi_{i}^{\mathrm{S}}$ is the surface pressure measured at time $t_{i}$. Once $J_{i}$ is known, we use eqn (5) in the form

$$
\Gamma=\frac{n}{n_{0}} \Gamma_{\text {app }}=\Gamma_{\text {app }} \exp (-J)
$$


In all cases, we did one or two iterations by using the results from Sec. 3.4 for $\tau_{\mathrm{d}}$, i.e.

(i) we used the first iteration for $\Gamma$ from eqn (7) to compute the solubility of the monolayer used in Sec. 3.4 to deal with the isobaric data and calculate the second iteration for $\tau_{\mathrm{d}}$ (the first being the one obtained from the 2-parameter fit, orange squares in Figure S13, and the second being the blue circles).

(ii) We used the second iteration for $\tau_{\mathrm{d}}$ to compute a new interpolation according to eqn (3), and use this interpolation in eqn (5) to produce a second iteration for $J$.

(iii) The new $J$ has been used to produce a second iteration for $\Gamma$ via eqn (7). The improvement from the iterations after the first was insignificant, even though the difference between the $\tau_{\mathrm{d}}$ in Figure S13 and the more accurate ones obtained in Sec. 3.4 is often large in the $\mathrm{LC}$ region.

Our previous procedure for solubility correction has been tested in ref. [17] by comparing the results from monolayer compression at different rates of motion of the barrier of the trough. We did a similar test with the new procedure. Two compression runs of dodecanol monolayer at $25{ }^{\circ} \mathrm{C}$ were performed, one at the highest possible velocity of the barrier, $270 \mathrm{~mm} / \mathrm{min}$, and the other at a 3 -fold lower speed of $90 \mathrm{~mm} / \mathrm{min}$. The results before and after the correction are illustrated in Figure S14. Before the correction, the slow isotherm is visibly shifted towards lower areas. After the correction, the two are in reasonable agreement, which proves the usefulness of the procedure. For the higher velocity, the correction leads to actual areas per molecule by 1-2 $\AA^{2}$ larger than the apparent areas (and less for lower temperatures). The figure also gives an idea of how bad the assumption for desorption under barrier control is: the slight misfit of the two curves is because of the overcorrection of the slower isotherm (where diffusion slows down the process additionally, which is not accounted for in the correction procedure). As seen, the difference is small enough to ignore (smaller than the reproducibility of the isotherms).

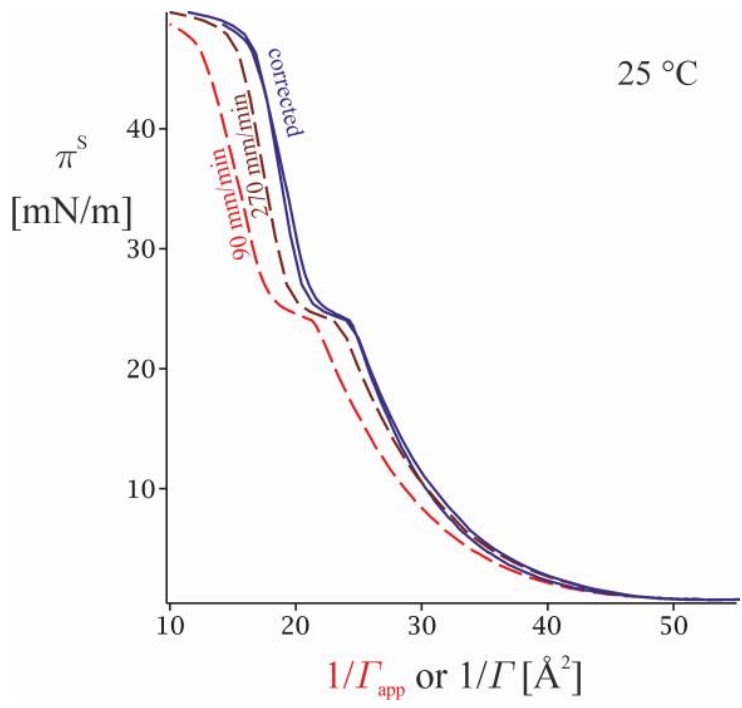


Figure S14. Surface tension vs. apparent area and corrected area per molecule of dodecanol spread on water at $25^{\circ} \mathrm{C}$ : data for two barrier velocities, 90 and $270 \mathrm{~mm} / \mathrm{min}$. The dashed lines are before the correction.

Our dodecanol isotherms can be compared with those of Fainerman et al. [50] at 10 and 15 ${ }^{\circ} \mathrm{C}$. Especially at $15{ }^{\circ} \mathrm{C}$, their results are shifted towards higher densities (Figure S15); the LC region is approaching unrealistic areas smaller than the crystallographic area of solid alkanes $\left(1 / \Gamma<18.2 \AA^{2}\right)$. This means that the data from [50] are significantly affected by solubility as well. In addition, the LE-LC phase transition of Fainerman et al. is at slightly higher surface pressure than ours. This can be explained either by the presence of impurities in theirs or our dodecanol, or by imperfect thermostating (the temperatures of Fainerman et al. appear to have been by $\sim 2^{\circ}$ higher than ours).
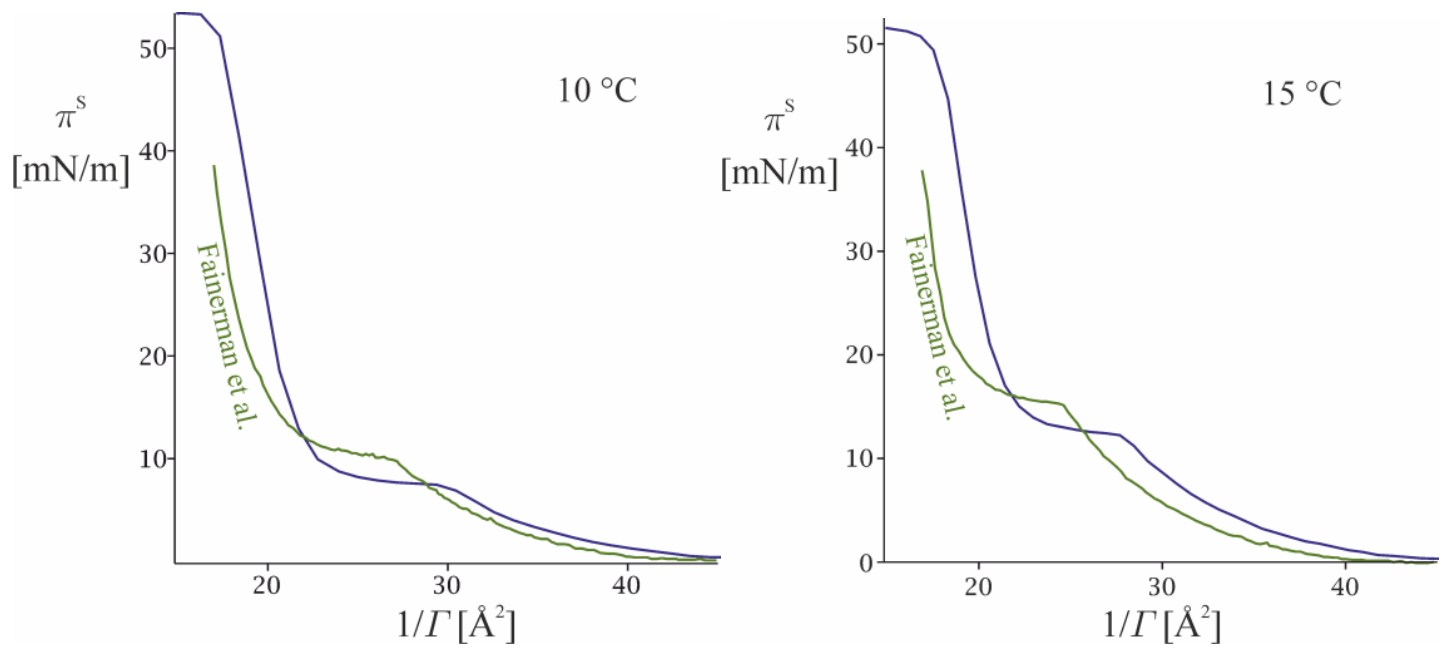

Figure S15. Comparison between our isotherms at 10 and $15^{\circ} \mathrm{C}$ and those by Fainerman et al. [50].

Kinetics of the LE-LC phase transition. Since we compress the monolayer quickly, we have to correct for the increased dynamic surface pressure in the phase transition region. Once the LC domains are formed, they start to interact repulsively with each other [51], and the force applied by the barrier on the heterogeneous monolayer is partly acting against this repulsive force (similar effects are common in the three dimensional liquid-solid phase transitions, when the fractal net of solid crystals in touch with each other start to have an elastic answer against the external force). The LC region covers a very short range of areas, and we usually have only 5-6 points in this region; the first few of them are affected by the dynamics of the phase transition, and the last few are affected by the collapse. In view of these complications, we decided that the crudest approximation for the equilibrium shape of the LC region - a line - is good enough. The following procedure was applied to all data:

(i) Identify the point at which the phase transition starts (indicated with an arrow in Figure S16); the data right of this point corresponds to homogeneous LE phase, while the data left of 
it refers to a heterogeneous surface with LC domains dispersed in an LE film (probably, the system relaxes to homogeneous LC monolayer eventually, but it is very likely that the LC domains and the two-dimensional LE films between them will survive even at significant compressions, close to the collapse).

(ii) The data in the LE region are fitted with eqn (16) $\left(\pi^{\mathrm{S}}>\pi_{\mathrm{pt}}^{\mathrm{S}}\right)-$ let the respective function be $f^{\mathrm{LE}}\left(\pi^{\mathrm{S}}\right)$. The data for the heterogeneous monolayer is fitted with a polynomial (of degree 3 or 4); we call the respective function $f^{\text {het }}\left(\pi^{\mathrm{S}}\right)$.

(iii) The point at which $f^{\mathrm{LE}}\left(\pi^{\mathrm{S}}\right)=f^{\text {het }}\left(\pi^{\mathrm{S}}\right)$ identifies the phase transition pressure tension $\pi_{\mathrm{pt}}^{\mathrm{S}}$ and the respective equilibrium $\mathrm{LE}$ adsorption $\Gamma^{\mathrm{LE}}$.

(iv) The data for the heterogeneous monolayer has an inflection. The tangent line through the inflection point of $f^{\text {het }}$ is constructed and is assumed to represent the equilibrium state of the LC monolayer (the equilibrium LC line in Figure S16).

(v) The point of cross-section of the equilibrium LC line and the horizontal line $\pi^{S}=\pi_{\mathrm{pt}}^{\mathrm{S}}$ defines the area of the LC monolayer in equilibrium with the LE film.

(vi) The value of the equilibrium spreading pressure $\pi_{\mathrm{pt}}^{\mathrm{S}}$ is substituted in the equation for the equilibrium LC line; this yields the area of the equilibrium spread layer.

This procedure is illustrated in detail in Figure S16, and also in Figure 2, in less detail. The edges of the horizontal dashed lines are part of the binodal for the LE-LC transition. It is remarkable that the slope of the LC region in Figure 2 (right) does not depend on the temperature significantly. The parameters of eqn (16) obtained via this procedure are summarized in Table 4.

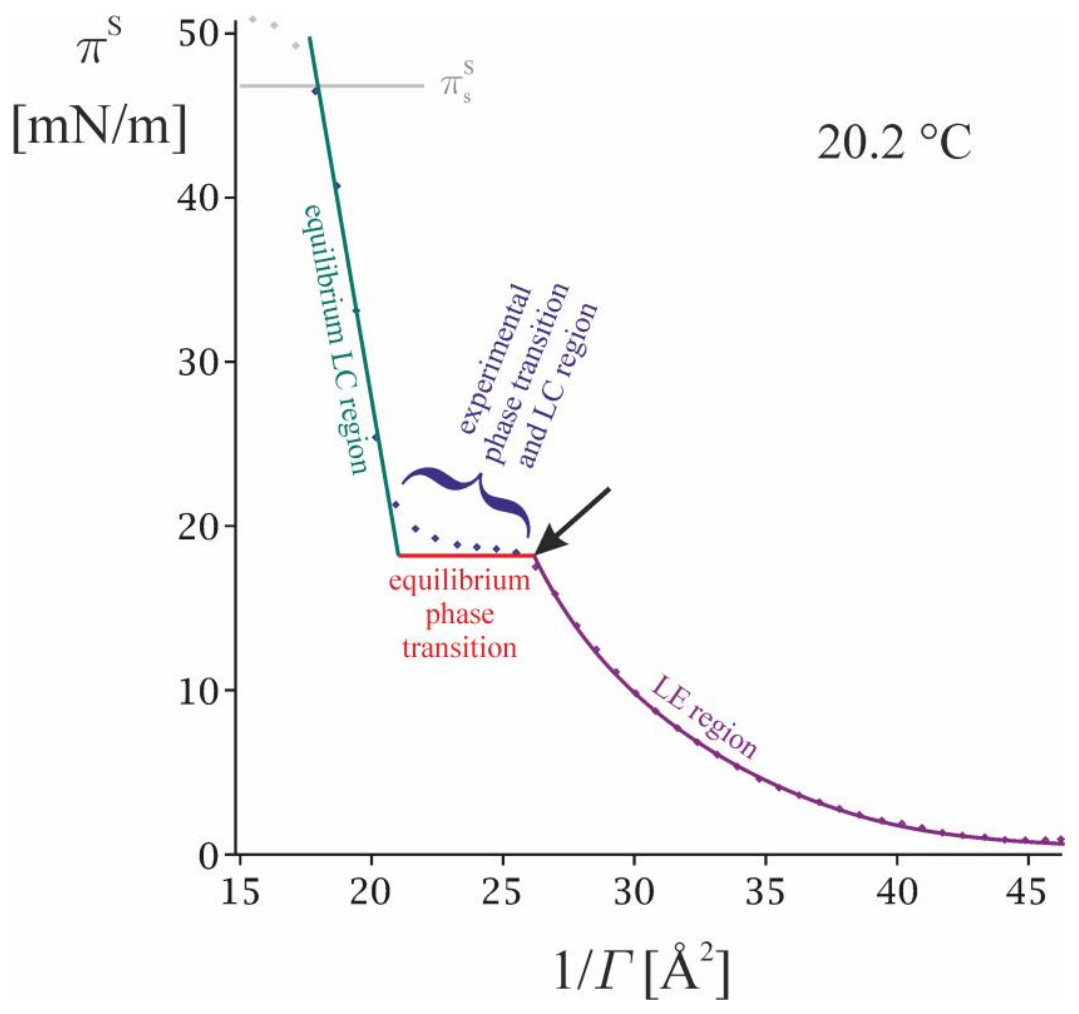


Figure S16. Correction for the kinetic effects during the LC-LE phase transition. The significant repulsion between the LC domains formed during the phase transition leads to a kinetic increase of the surface pressure $\pi^{\mathrm{S}}$. The observed $\pi^{\mathrm{S}}$ vs. $\Gamma^{-1}$ curve in this region is therefore below the theoretically expected horizontal line for a first order phase transition.

Table 4. Parameters of the equation of state (16), as obtained from the corrected $\pi^{\mathrm{S}}$ vs. $1 / \Gamma$ isotherms.

\begin{tabular}{|r|c|c|c|c|c|c|}
\hline $\boldsymbol{T}$ & $\mathbf{1 0}{ }^{\circ} \mathbf{C}$ & $\mathbf{1 5 . 4}{ }^{\circ} \mathbf{C}$ & $\mathbf{1 7}^{\circ} \mathbf{C}$ & $\mathbf{2 0 . 2}{ }^{\circ} \mathbf{C}$ & $\mathbf{2 2 . 9}{ }^{\circ} \mathbf{C}$ & $\mathbf{2 5}^{\circ} \mathbf{C}$ \\
\hline$g_{1}\left[\AA^{2}\right]$ & 24.2836 & 30.4535 & 31.4014 & 22.2778 & 23.07556 & 14.2358 \\
\hline$g_{2}$ & -0.0269519 & -0.238060 & -0.238816 & -0.134695 & -0.121569 & -0.0585756 \\
\hline$g_{3}$ & -0.972393 & -0.758748 & -0.755349 & -0.859611 & -0.872810 & -0.934531 \\
\hline$m_{1}$ & 1 & 5 & 4 & 2 & 2 & 1 \\
\hline$m_{2}$ & 30 & 19 & 15 & 14 & 14 & 19 \\
\hline$m_{3}$ & 1 & 3 & 2 & 2 & 2 & 2 \\
\hline$g_{4}\left[\AA^{2}\right]$ & 22.5770 & 22.2171 & 22.1276 & 22.9717 & 23.6772 & 24.8072 \\
\hline$g_{5}\left[\AA^{2} \mathrm{~m} / \mathrm{mN}\right]$ & 0.0978461 & 0.0875110 & 0.0762142 & 0.107170 & 0.105664 & 0.146501 \\
\hline$\pi_{\mathrm{pt}}^{\mathrm{S}}[\mathrm{mN} / \mathrm{m}]$ & 7.38686 & 12.1554 & 14.5308 & 18.1982 & 20.8908 & 23.8605 \\
\hline$a_{\mathrm{pt}}^{\mathrm{LE}}\left[\AA^{2}\right]$ & 30.07 & 28.2120 & 27.0654 & 26.1812 & 26.0406 & 25.0290 \\
\hline$a_{\mathrm{pt}}^{\mathrm{LC}}\left[\AA^{2}\right]$ & 21.85 & 21.1534 & 21.0201 & 21.0214 & 21.4697 & 21.3116 \\
\hline$a_{\mathrm{collapse}}\left[\AA^{2}\right]$ & 18.00 & 18.12 & 18.56 & 17.96 & 18.73 & 17.9492 \\
\hline
\end{tabular}

Figure S17 illustrates the computation via the Gibbs equation (3) of the chemical potential $\Delta_{\mathrm{s}} \mu=\mu-\mu_{\mathrm{s}}$ from the isotherm fits just discussed. The state of the surfactant in the crystal is used as a standard state - the integration starts at the spreading pressure of dodecanol crystals.

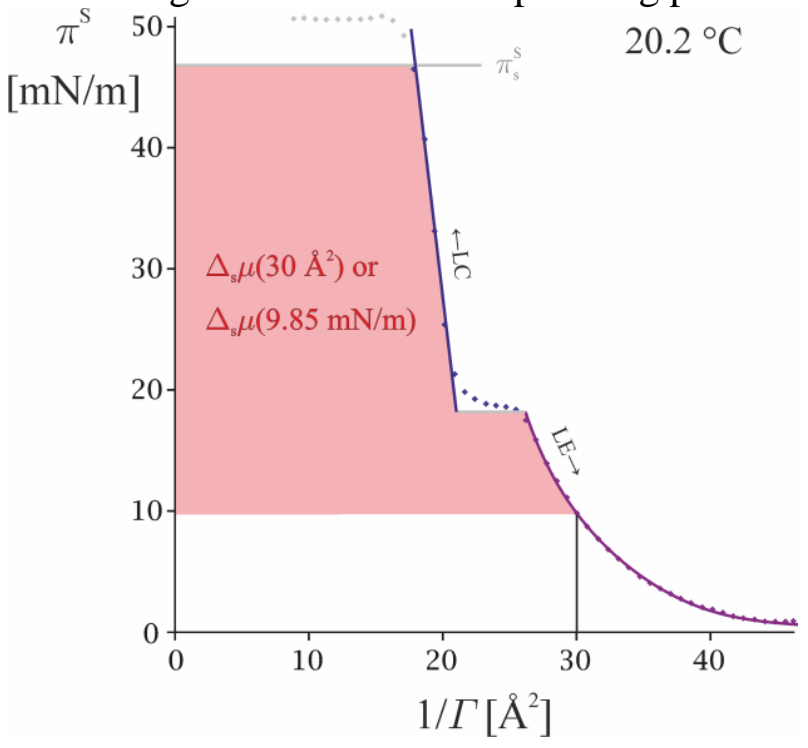

Figure S17. Graphical representation of the chemical potential $\Delta_{\mathrm{s}} \mu$. The surface pressure vs. area isotherm at $20.2{ }^{\circ} \mathrm{C}$ is used to compute the value of $\Delta_{\mathrm{s}} \mu$ at $\pi^{\mathrm{S}}=9.85 \mathrm{mN} / \mathrm{m}$ (corresponding to $1 / \Gamma=30$ 
$\AA^{2}$ ). According to eqn (3), $\Delta_{\mathrm{s}} \mu$ is given by minus the shaded area. $\pi_{\mathrm{s}}^{\mathrm{S}}$ is the spreading pressure of dodecanol crystals.

For the sake of completeness and for future reference, we also analysed the data for the LE-LC phase transition pressure $\pi_{\mathrm{pt}}^{\mathrm{S}}$ as a function of temperature. Unlike the area per molecule, the surface pressure of the phase transition is unaffected by the desorption and the leakages, so we collected a significant amount of data in the range $10-30{ }^{\circ} \mathrm{C}$, Figure S18. The line in this figure is a quadratic fit. Using the data for $\pi_{\mathrm{pt}}^{\mathrm{S}}$ from Figure S18 and the phase transition areas $a_{\mathrm{pt}}^{\mathrm{LE}}$ and $a_{\mathrm{pt}}^{\mathrm{LC}}$ from Table 4 , we were able to compute the heat of the phase transition $\Delta h_{\mathrm{pt}}$ through the 2D-Clausius-Clapeyron equation,

$$
\frac{\mathrm{d} \pi_{\mathrm{pt}}^{\mathrm{S}}}{\mathrm{d} T}=\frac{\Delta h_{\mathrm{pt}}}{T\left(a_{\mathrm{pt}}^{\mathrm{LE}}-a_{\mathrm{pt}}^{\mathrm{LC}}\right)} .
$$

The dependence of $\Delta h_{\mathrm{pt}}$ on $T$ that follows from this equation is illustrated in Figure S19.

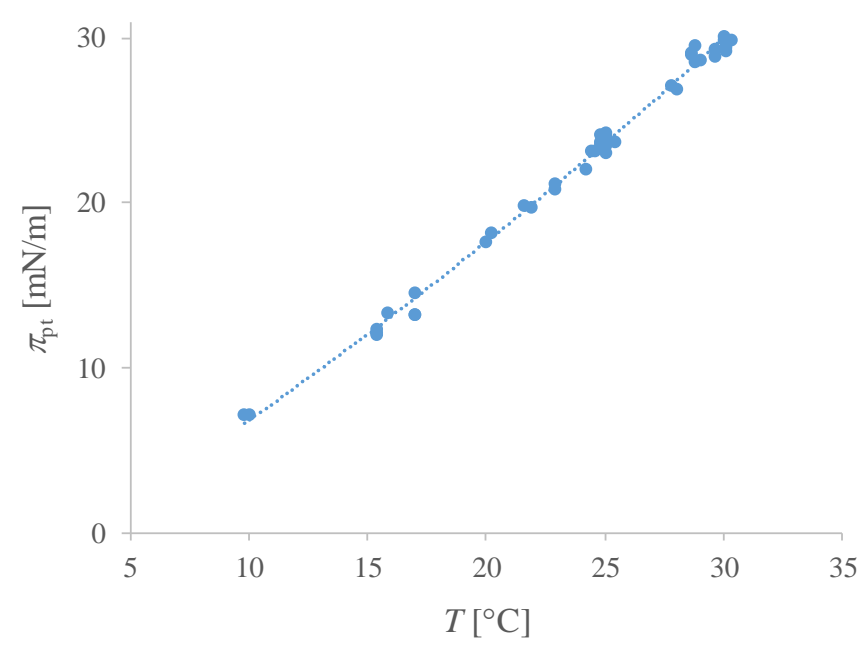

Figure S18. Surface pressure of the LE-LC phase transition as a function of the temperature. 


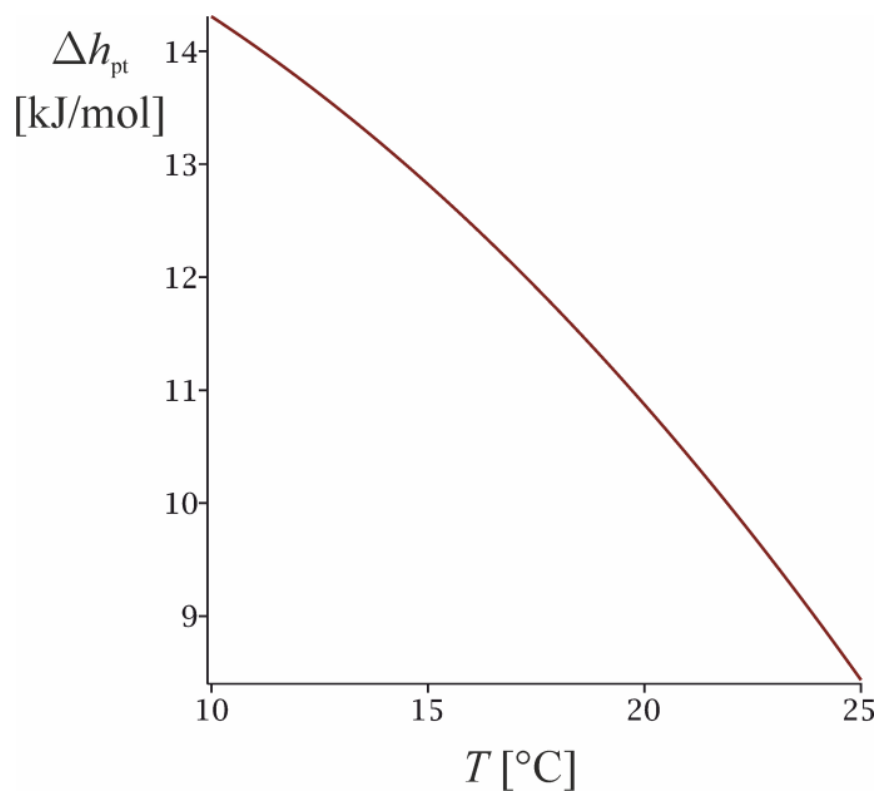

Figure S19. Heat of the LE-LC phase transition.

\section{S5. Leakage through the barrier}

The test involved placement of dodecanol crystals in one compartment of the trough and measurement of the surface tension in the other (which should remain clean in the absence of leakage, $\sigma=\sigma_{0}$ ), with a barrier separating the two compartments. In the first run (the upper curve in Figure S20), leakage was negligible for 4 minutes, then it caused the observed decrease of $\sigma$ in the crystal-free compartment. In the second run (lower curve), leakage was immediately apparent. The plateau reached at the $3^{\text {rd }}$ minute corresponds to the LE-LC phase transition.

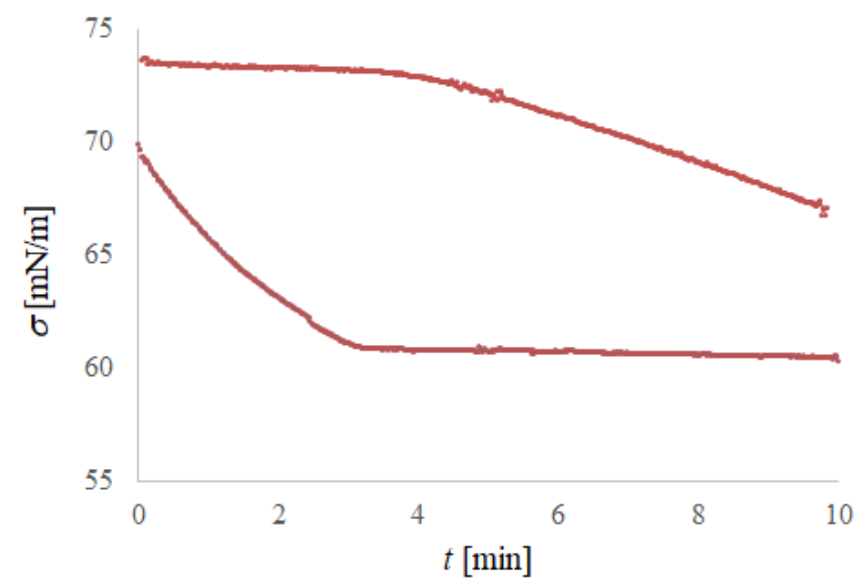

Figure S20. An illustration of random leakage through the movable barrier: in both runs, crystallites are placed in one of the compartments to form an equilibrium spread monolayer at $16{ }^{\circ} \mathrm{C}$. The surface 
tension in the second compartment (the surface of which is initially alcohol-free) is measured as a function of time.

\section{S6. Dodecanol - additional data}

Here, we present most of our desorption isobars at temperatures from 10 to $23{ }^{\circ} \mathrm{C}$ (Figure $\mathrm{S} 21$, points). These are compared to the theoretical model (13), with value of $\tau_{\mathrm{d}}$ from eqn (24)-(25) and value of $\tau_{\text {tr }}$ from eqn (11), with the parameters from Table 2.
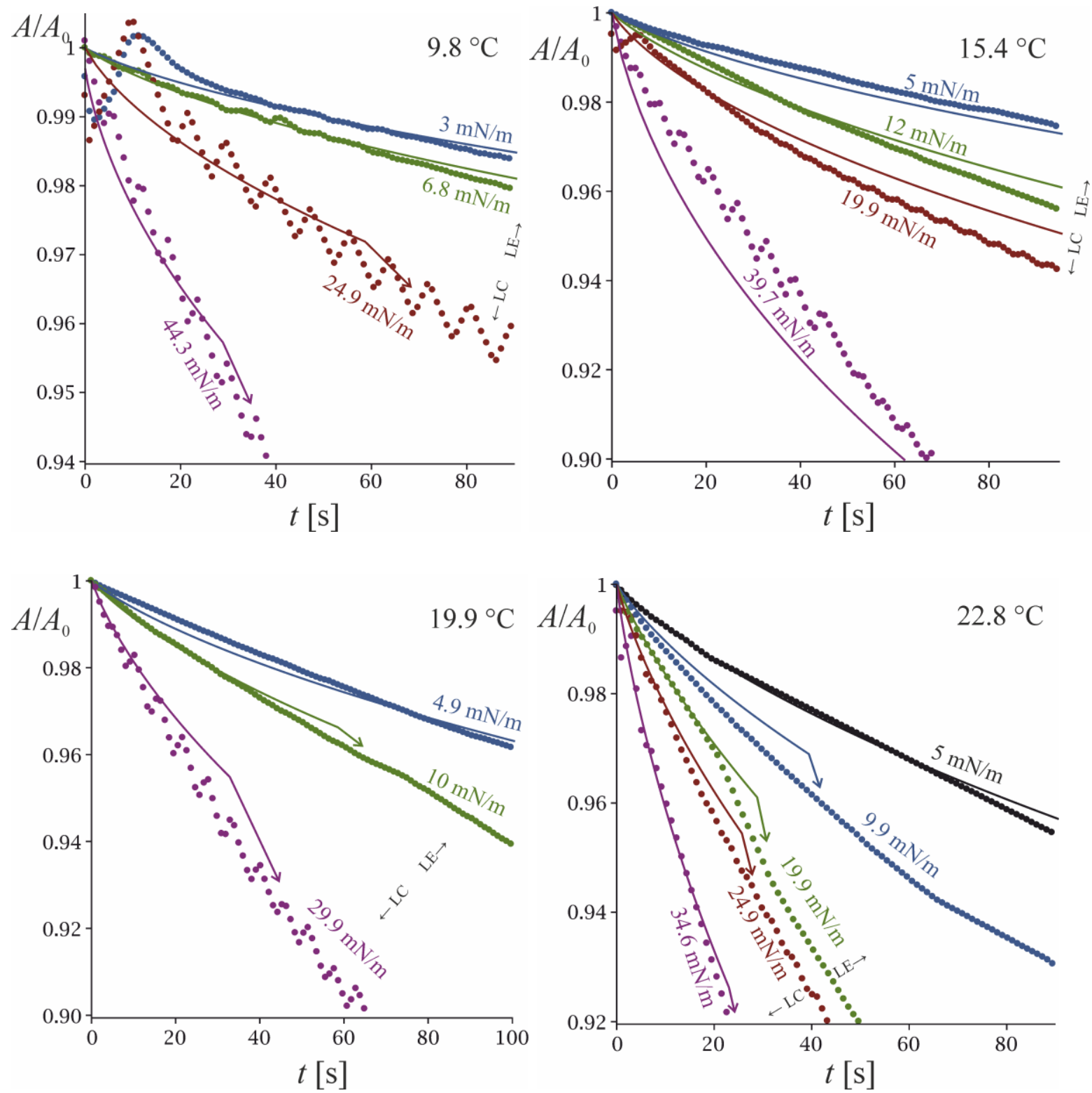
Figure S21. Desorption isobars (relative decrement of the area vs. time) at several fixed surface pressures and temperatures. Solid lines: the theoretical prediction (13) for mixed barrier-diffusion control, with desorption time $\tau_{\mathrm{d}}$ linear with $1 / \Gamma$ (eqn (24) with $K^{\mathrm{LE}}$ and $K^{\mathrm{LC}}$ from Table 2).

At higher temperatures $\left(25-30{ }^{\circ} \mathrm{C}\right)$, the desorption is under barrier control as in Figure 9. However, due to the leakages, we were unable to measure the adsorption isotherms at 29 and $30{ }^{\circ} \mathrm{C}$, although we measured desorption isobars. Therefore, in Figure S22, the measured isobars are presented without comparison to theory. We attempted to estimate the required quantities ( $\tau_{\mathrm{d}}$ and $\Gamma$ ) by extrapolation of the $\pi^{\mathrm{S}}$ vs. $1 / \Gamma$ isotherms in Figure 2 and the $\tau_{\mathrm{d}}$ formulae (24)-(25) (which are strictly valid in the range $10-25^{\circ} \mathrm{C}$ ). Overall, the observed desorption rates seem to be slightly faster than the predicted ones from this extrapolation (as if the real $\tau_{\mathrm{d}}$ at 30 ${ }^{\circ} \mathrm{C}$ is by $c a .25 \%$ smaller than the extrapolated). This might mean that the activation energy $E_{\mathrm{A}}^{\mathrm{LE}}$ is underestimated, but might also be due to leakages, evaporation or, in part, to inaccurate extrapolation of the compression isotherm. 

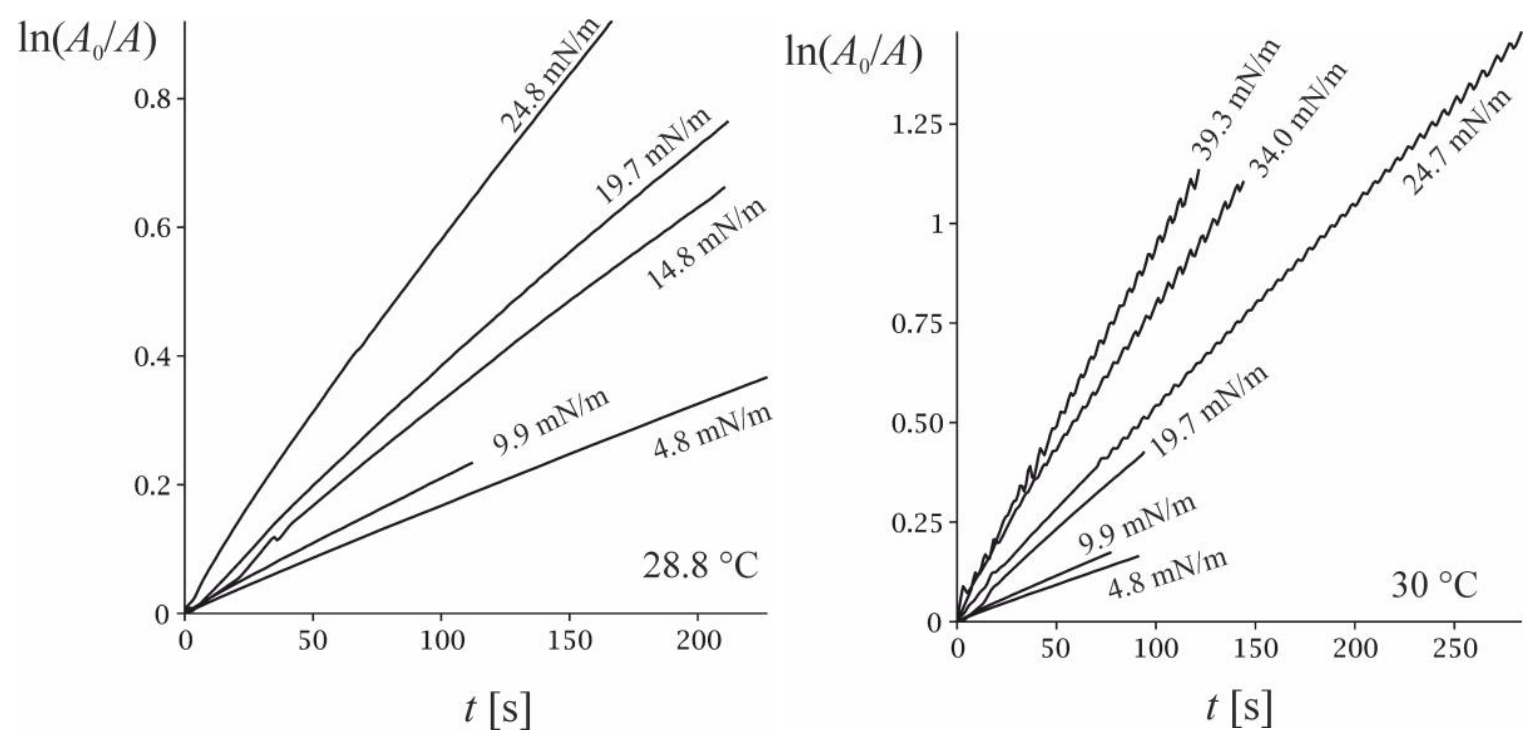

Figure S22. Desorption isobars at $29-30{ }^{\circ} \mathrm{C}$ and several fixed surface pressures. The process seems to proceed under barrier control (similarly to the data at $25^{\circ} \mathrm{C}$ in Figure 9) for at least 50-100 s, but in the absence of compression isotherm data, we did not try to interpret these data.

In Figure 23, the data of De Keyser and Joos for dodecanol is compared with the theoretical prediction for desorption under pure barrier control (dashed line) and desorption in the $\tau_{\mathrm{d}}-D$ regime (solid line). As discussed in sec. 4, in the time range of interest to us (0-200 s), the data indeed suggests barrier control, exactly as our measurements at $25^{\circ} \mathrm{C}$ (Figure 9).

$$
t[\mathrm{~s}]
$$

Figure 23. Desorption isobar by De Keyser and Joos at $8 \mathrm{mN} / \mathrm{m}$ (probably $25^{\circ} \mathrm{C}$ ). Solid line: the theoretical prediction (13) for mixed barrier-diffusion control. Dashed line: pure barrier control, eqn (14). The adsorption $\Gamma$ and the desorption time $\tau_{\mathrm{d}}$ at $8 \mathrm{mN} / \mathrm{m}$ were calculated from eqn (16)\&(24).

Figure S24 illustrates the dispersion analysis of the fit of the $\tau_{\mathrm{d}}$ data in Table 2 with eqn (24)-(25). All values of the parameters $K_{0}$ and $E_{\mathrm{A}}$ that fall inside the ellipses " $1.05 \times d e v_{\min }^{2}$ " will produce dispersion by $5 \%$ larger than the minimal. 

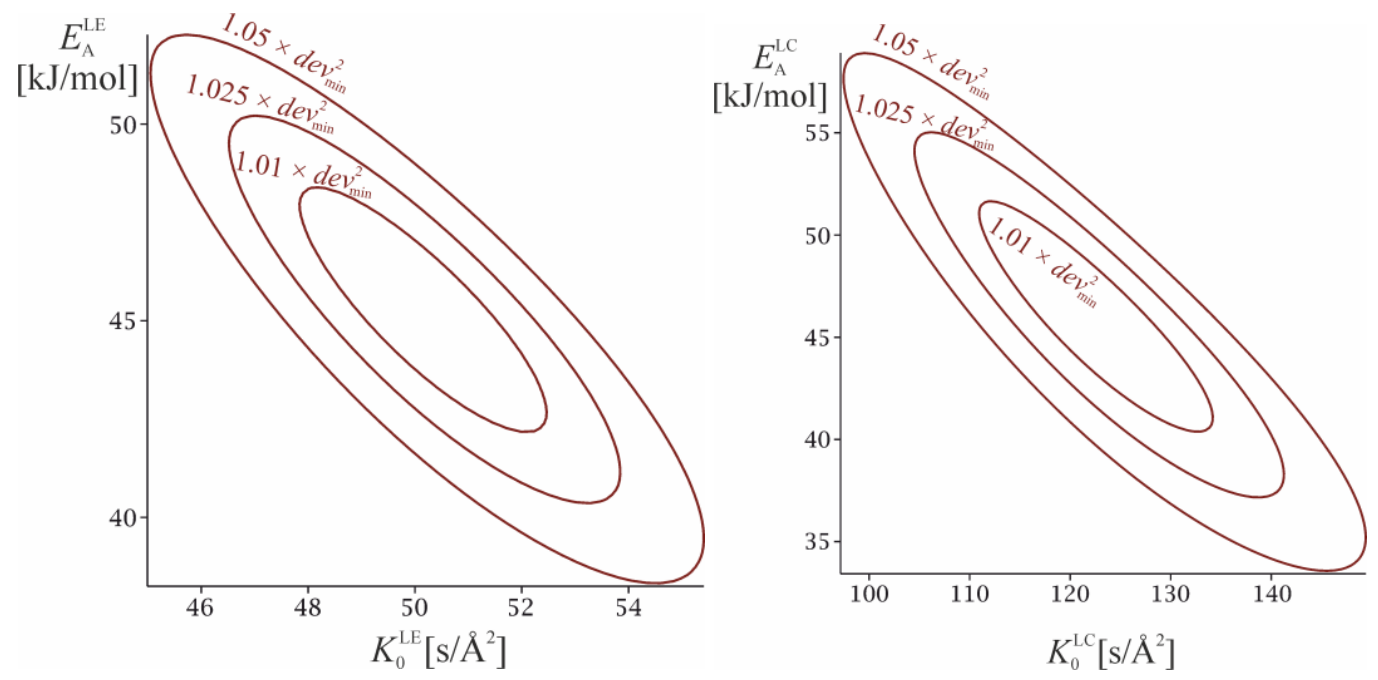

Figure S24. Contour plot of the dispersion of eqn (24)-(25) against the $\tau_{\mathrm{d}}$ data in Table 2.

\section{S7. Decanol data}

The sticky disc adsorption model predicts a theoretical surface pressure vs. concentration isotherm $\pi_{\mathrm{th}}^{\mathrm{S}}\left(C_{\mathrm{eq}} ; K_{\mathrm{a}}, \alpha, \beta\right)$ (a numerical solution to eqn (18)-(19)\&(4)). The value of the harddisc area $\alpha$ is set to the one following from crystallographic data, $16.5 \AA^{2}$. The other two parameters are obtained through minimization of the merit function

$$
\operatorname{dev}_{\pi}^{2}\left(K_{\mathrm{a}}, \beta\right)=\frac{1}{N-2} \sum_{i}\left[\pi_{\mathrm{th}}^{\mathrm{s}}\left(C_{i} ; K_{\mathrm{a}}, \beta\right)-\pi_{i}^{\mathrm{s}}\right]^{2} .
$$

Here, $C_{i}$ and $\pi_{i}^{\mathrm{S}}$ are the experimental surfactant concentrations and surface pressures from ref. $[29,38,39,63,82]$. We used only low pressure data with $\pi^{\mathrm{S}}<30 \mathrm{mN} / \mathrm{m}$, since it is likely that at higher surface pressures the monolayer is in the LC state where eqn (18) is invalid, and anyway, the data of Baret et al. are in the range $5-20 \mathrm{mN} / \mathrm{m}$. The result of the regression for the best fit parameters is $\beta=20.7$ and $K_{\mathrm{a}}=39.25 \mu \mathrm{m}$, i.e. $\ln \left(K_{\mathrm{a}} /[\mathrm{m}]\right)=-10.15$. These can be compared to $\beta=14$ and $\ln \left(K_{\mathrm{a}} /[\mathrm{m}]\right)=-9.8$ from ref. [60] (the latter values are not accurate enough for longchained alcohols such as decanol). The comparison between the data points and the theoretical sticky disc curve with the best fit parameters is shown in Figure S25.

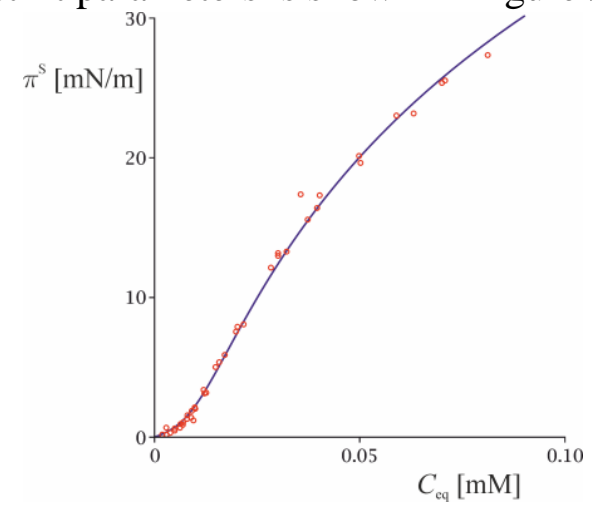

Figure S25. Adsorption isotherm of decanol: data from ref. $[29,38,39,63,82]$ and the best fit with the sticky disc model (18)-(19)\&(4), with $K_{\mathrm{a}}=39.25 \mu \mathrm{m}$ and $\beta=20.7$. 
The values obtained for $K_{\mathrm{a}}$ and $\beta$ allow the computation of $\Gamma$ and $\gamma^{\mathrm{S}}$ at each surface pressure of Baret et al. We calculated the adsorptions by solving eqn (18) for $\Gamma$; this value of $\Gamma$ was further substituted in eqn (19) to find the respective surface activity coefficient $\gamma^{\mathrm{S}}$. Eqn (4) was then used to calculate $C_{\text {eq. }}$. The results for $\Gamma$ and $C_{\text {eq }}$ at the four surface pressures of Baret at al. are given in Table 3 in the main text.

This leaves a single unknown parameter in eqn (13) for the kinetics of desorption: the desorption rate constant $\tau_{\mathrm{d}}$. To find $\tau_{\mathrm{d}}$, eqn (13) has been used to fit the $A$ vs. $t$ data of Baret et al. [6] at each surface pressure. The results are illustrated in Figure S26, and the values of $\tau_{\mathrm{d}}$ are summarized in Table 3. An acceleration of the diffusion process is evident at the longest times $\left(t>t_{\max }\right.$ ) for $\pi^{\mathrm{S}}=5,10$ and $15 \mathrm{mN} / \mathrm{m}$ (discussed also by Baret et al. [6]) - therefore, only the data at $t<t_{\max }$ were used for the regression. The times $t_{\max }$ where first signs of convective regime are seen were determined iteratively using positive deviations from eqn (13) as a criterion, similarly to the procedure for dodecanol in sec. 3.4. The time of transition is marked with red dots in Figure S26.

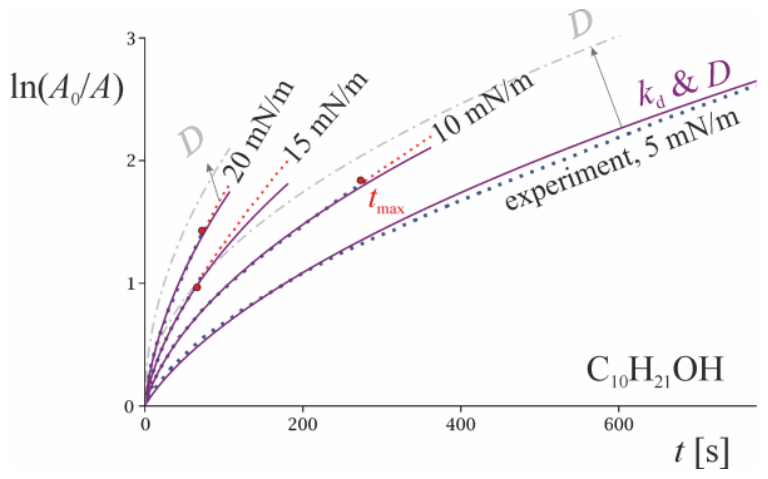

Figure S26. $\ln \left(A_{0} / A\right)$ vs $t$ [s] for $\mathrm{C}_{10} \mathrm{H}_{21} \mathrm{OH}$ - data from ref. [6] (dot lines) at 4 surface pressures (5-20 $\mathrm{mN} / \mathrm{m}$ ). Dash-dot lines: $D$-regime, eqn (15), overpredicting the dissolution rate compared to the experiment. Solid lines: fits to the experimental data of eqn (13) for the mixed barrier-diffusion mechanism $\left(\tau_{\mathrm{d}} \& D\right)$. The best-fit values of $\tau_{\mathrm{d}}$ are listed in Table 3. Acceleration of the desorption process due to convection is evident above certain $t_{\max }$ (marked with circles); all data after $t>t_{\max }$ were ignored for the fit. 